\title{
Impact of Grape Variety, Yeast and Malolactic Fermentation on Volatile Compounds and Fourier Transform Infrared Spectra in Red Wines
}

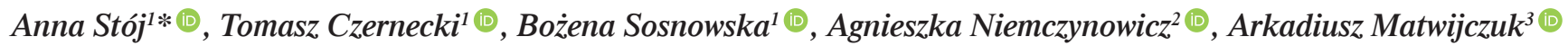 \\ ${ }^{1}$ Department of Biotechnology, Microbiology and Human Nutrition, Faculty of Food Science and Biotechnology, \\ University of Life Sciences in Lublin, Skromna 8, 20-704 Lublin, Poland \\ ${ }^{2}$ Faculty of Mathematics and Computer Science, University of Warmia and Mazury in Olsztyn, Stoneczna 54, 10-710 Olsztyn \\ ${ }^{3}$ Department of Biophysics, University of Life Sciences in Lublin, Akademicka 13, 20-950 Lublin
}

Key words: fermentation, Saccharomyces cerevisiae, Saccharomyces bayanus, Oenococcus oeni, aroma compounds, Fourier transform infrared spectroscopy

Volatile compounds are very important to the flavour and quality of the wine. The study aimed to determine the effect of grape variety (Rondo and Zweigelt), yeast, malolactic fermentation (MLF) and yeast $\times$ MLF interaction on the content of volatile compounds in red wines. The wines were produced by sequential inoculation with five commercial yeast strains and a commercial lactic acid bacteria (LAB) strain (induced malolactic fermentation) as well as by inoculation with five commercial yeast strains and without LAB inoculation (spontaneous malolactic fermentation). The volatile compounds were determined by headspace solid-phase microextraction/gas chromatography-mass spectrometry (HS-SPME-GC/MS). Forty-six volatile compounds belonging to alcohols, esters, acids, aldehydes, ketones, furan compounds, sulfur compounds and volatile phenols were identified in the wines. The grape variety was the factor with a significant impact on the highest number of volatile compounds, 32 out of 46 . Furthermore, 7 compounds were affected by yeast, 10 by MLF and only 3 by yeast $\times$ MLF interaction. Characteristic bands in Fourier transform infrared (FTIR) spectra were assigned to the vibrations of functional groups of volatile compounds. The whole FTIR spectra were analysed in detail; three characteristic spectral ranges such as 3650-2700, 1750-1500, and below $1500 \mathrm{~cm}^{-1}$ were shown for different classes of volatile compounds. The most remarkable spectral changes were observed for the last two areas.

\section{INTRODUCTION}

Volatile compounds (VOCs) largely determine the aroma of the wine. The concentration of VOCs in wines ranges from several $\mathrm{mg} / \mathrm{L}$ to a few ng/L [Welke et al., 2012; Zhu et al., 2016]. These volatile compounds originate from grapes (varietal aromas) and are secondary products of fermentation processes (fermentation aromas) and aging (post-fermentation aromas) [Callejon et al., 2010]. Alcoholic fermentation (AF) and malolactic fermentation (MLF) processes play a fundamental role in creating the taste and aroma of the product [Gammacurta et al., 2014, 2017].

Yeasts, responsible for AF, transform sugar into ethanol, carbon dioxide, and minor secondary metabolites, including higher alcohols, esters, fatty acids, aldehydes, ketones, and others [Englezos et al., 2018]. Yeasts also release varietal aromatic compounds from grapes. The ability to synthesize secondary metabolites and to release varietal compounds depends on the yeast species and strain [Blanco et al., 2014; Callejon et al., 2010; Gammacurta et al., 2017]. The primary yeast species responsible for AF is Saccharomyces cerevisiae [Azzolini et al.,
2012]. A wide range of selected yeast strains are commercially available that guarantee fermentation control and wine quality. However, some wineries are interested in selecting autochthonous (indigenous) yeast starters because of their ability to ensure the sensory characteristic of wines originating from a specific terroir [Blanco et al., 2014; Tufariello et al., 2014].

During MLF, L-malic acid is an enzymatically decarboxylated into L-lactic acid and carbon dioxide by lactic acid bacteria (LAB), mainly Oenococcus oeni [Tristezza et al., 2016]. This process is an important step in the production of most red and some white and sparkling wines [Abrahamse \& Bartowsky, 2012; Costello et al., 2012; Lasik-Kurdyś et al., 2018]. MLF reduces the total acidity (by slightly increasing the $\mathrm{pH}$ ), causes microbiological stability, and modifies wine aroma and taste [Costello et al., 2012]. Aroma modification is associated with citric acid catabolism by $\mathrm{LAB}$, resulting in the production of diacetyl, acetic acid, acetoin, and 2,3-butanediol [Malherbe et al., 2012]. MLF typically takes place after AF and can occur spontaneously, carried out by indigenous LAB populations, or may be induced by starter culture inoculation to control this step of winemaking. Two types of LAB

\footnotetext{
* Corresponding Author:

E-mail: anna.stoj@up.lublin.pl (A. Stój)
} 
inoculation are commonly used: traditional inoculation after $\mathrm{AF}$ (sequential) or simultaneous inoculation with the yeast and LAB (co-inoculation) [Abrahamse \& Bartowsky, 2012; Antalick et al., 2013]. The undoubted advantage of simultaneous inoculation is the reduction of the vinification time. However, this inoculation may slow down the growth and deteriorate the viability of yeast cells, leading to a delay or inhibition of AF, and increase volatile acidity caused by higher acetic acid production [Stój, 2020a].

In recent years, several authors have conducted studies on the influence of grape variety and microorganisms on the content of volatile compounds in red wines [Cañas et al., 2012; Cioch-Skoneczny et al., 2021; Englezos et al., 2018; Tristezza et al., 2016]. The grape variety affects not only varietal aromas (C13-norisoprenoids, lactones, and terpenes) but also other volatile compounds, such as higher alcohols, esters, and fatty acids [Vilanova et al., 2007]. Both grape cultivar and yeast species significantly impacted content of ethyl esters in wines. The effect of grape cultivar on the content of higher alcohols seems more significant than the effect of yeast species. Fatty acid (acetic acid, 2-methylpropanoic acid, butanoic acid, 3-methylbutanoic acid and octanoic acid) contents showed significant differences as an effect of yeast species [Liu et al., 2017]. A specific volatile profile corresponded to each yeast/ LAB combination, wherein the yeast strain had a predominant effect on aromatic compounds [Gammacurta et al., 2017]. The wine matrix, particularly pre-MLF $\mathrm{pH}$, ethanol content, and grape source affected the ability of LAB strains to modulate volatile compounds in wines [Costello et al., 2012].

The composition of grapes depends on the varietal and clonal genotype of the grapevine as well as the interaction of the genotype and phenotype with many environmental factors (terroir) [Styger et al., 2011]. One of the most popular red grape hybrids in Poland is Rondo (non-Vitis vinifera). It derives from a cross between two species of Vitis, Zarya severa $\times$ Saint Laurent [Wojdyło et al., 2018]. However, noble grape varieties, such as Zweigelt (Vitis vinifera), cover a small area of Poland [Stój et al., 2020b]. To the best of our knowledge, there have been no studies on the varieties of grapes cultivated in Poland and the wine factors influencing the concentration of different classes of volatile compounds in red wines. The study aimed to determine the effects of Rondo and Zweigelt varieties, yeast, MLF, and yeast $\times$ MLF interaction on the content of volatile compounds in wines. The wines were produced by sequential inoculation of grape pulp with five commercial yeast strains (S. cerevisiae or $S$. cerevisiae $\times$ $S$. bayanus) and a commercial LAB $(O$. oeni) as well as by inoculation with five commercial yeast strains and without LAB inoculation. Moreover, the analysis of Fourier transform infrared (FTIR) spectra used in conjunction with the multivariate analysis allowed for assigning vibration bands to specific functional groups of volatile compounds.

\section{MATERIALS AND METHODS}

\section{Winemaking}

Details of winemaking are presented in our previous article [Stój et al., 2020b]. The grapes of Zweigelt and Rondo varieties were obtained from 'Małe Dobre' and 'Dom
Bliskowice' vineyards, respectively. The vineyards are located in the Lublin Province, Poland. The grapes were harvested manually in 2017. The grape pulp was subjected to alcoholic fermentation by using five commercial yeast strains, four S. cerevisiae: SafOEno ${ }^{\mathrm{TM}}$ SC 22, Essentiale Grand Cru (Lesaffre, Marcq-en-Baroul, France), Siha Active Yeast 8, Siha Rubino Cru (Eaton, Tinton Falls, NJ, USA); and one S. cerevisiae $\times S$. bayanus - SafOEno ${ }^{\mathrm{TM}}$ HD S62 (Lesaffre), the same for the Zweigelt and Rondo varieties. Spontaneous MLF occurred in one part of the wines, i.e. that without LAB inoculation, and induced MLF - in the other part of the wines, i.e. that with $O$. oeni (Viniflora Oenos, Eaton) inoculation. O. oeni starter culture was added after the completion of AF (sequential inoculation) to the wines in which induced malolactic fermentation was carried out. We did not obtain complete reduction of malic acid in any of the wines. The concentrations of malic and lactic acids in the final wines are presented in the supplementary material to the previous article [Stój et al., 2020b]. The experiments were performed in duplicate. The following abbreviations are used for the wines: Z1-Z5 - Zweigelt wines, in which AF was induced using various yeast strains, and the wines were left to undergo spontaneous MLF; Z1 LAB-Z5 LAB - Zweigelt wines, in which $\mathrm{AF}$ was induced using various yeast strains (the same strains as in Z1-Z5 wines), and MLF was carried out by inoculation with lactic acid bacteria; R1-R5 - Rondo wines, in which AF was induced using various yeast strains, and the wines were left to undergo spontaneous MLF; R1 LAB-R5 LAB - Rondo wines, in which $\mathrm{AF}$ was induced using various yeast strains (the same strains as in R1-R5 wines), and MLF was carried out by inoculation with lactic acid bacteria.

\section{Reagents}

Sodium chloride and hydrochloric acid were obtained from POCh (Gliwice, Poland). Sodium chloride was oven-dried at $200^{\circ} \mathrm{C}$ overnight. Hydrochloric acid was dissolved in water at a concentration of $78 \mathrm{~g} / \mathrm{L}$. 4-Hydroxy-4-methyl-2-pentanone (the internal standard of volatile compound analysis) was purchased from Sigma-Aldrich (Saint Louis, MO, USA) and prepared in water at a concentration of $7 \mathrm{mg} / \mathrm{L}$. A mixture of $n$-alkanes $\left(\mathrm{C}_{7}-\mathrm{C}_{30}\right)$ for the calculations of linear retention indices (RI) was supplied by Supelco (Bellefonte, PA, USA). All chemicals were of analytical grade.

\section{Determination of volatile compounds}

The volatile compounds were determined by headspace solid-phase microextraction and gas chromatography-mass spectrometry analysis (HS-SPME-GC/MS) according to our previous method [Stój et al., 2017a,b] with slight modification.

A fiber holder and an $85 \mu \mathrm{m}$ CAR/PDMS fiber were used (Supelco). Before each analysis, the fiber was conditioned by inserting it into the auxiliary $\mathrm{GC}$ injection port at $280^{\circ} \mathrm{C}$ for $5 \mathrm{~min}$. Then, $0.9 \mathrm{~g}$ of $\mathrm{NaCl}, 3 \mathrm{~mL}$ of wine, $50 \mu \mathrm{L}$ of $\mathrm{HCl}, 100 \mu \mathrm{L}$ of 4-hydroxy-4-methyl-2-pentanone, and a magnetic stirring bar were placed in a glass vial of $7 \mathrm{~mL}$. The vial was capped with a PTFE-silicone septum (Supelco) on which a screw cap with a hole was placed. The vial was placed in a block on an MS7-H550-S magnetic stirrer with a hotplate (DLAB Scientific Co., Beijing, China). The sample was incubated at $40^{\circ} \mathrm{C}$ 
for $15 \mathrm{~min}$, and then the fiber was exposed to the headspace (HS) at $40^{\circ} \mathrm{C}$ for $30 \mathrm{~min}$. The incubation and microextraction were carried out with continuous stirring at a minimum speed. The fiber was thermally desorbed in the GC injection port for $2 \mathrm{~min}$ at $220^{\circ} \mathrm{C}$ in the split-less mode.

The samples were analyzed using a gas chromatograph combined with a quadrupole mass spectrometer (GCMS-QP2010, Shimadzu, Kyoto, Japan). All analyses were made in triplicate. Chromatographic separations were carried out using a VF-WAXms capillary column $(60 \mathrm{~m}, 0.25 \mathrm{~mm}$ ID, $0.25 \mu \mathrm{m}$ film thickness, $100 \%$ polyethylene glycol, Agilent, Santa Clara, CA, USA). Helium was used as the carrier gas at a flow rate of $1.8 \mathrm{~mL} / \mathrm{min}$. The column oven temperature was held at $34^{\circ} \mathrm{C}$ for $5 \mathrm{~min}$, then raised to $100^{\circ} \mathrm{C}$ at a rate of $3^{\circ} \mathrm{C} / \mathrm{min}$ and held for $6 \mathrm{~min}$, and finally raised to $220^{\circ} \mathrm{C}$ at a rate of $5^{\circ} \mathrm{C} / \mathrm{min}$ and held for $15 \mathrm{~min}$. The total run time was $72 \mathrm{~min}$. The specification of the mass spectrometer was as follows: electron ionization source with a temperature of $200^{\circ} \mathrm{C}, 70 \mathrm{eV}$ ionization energy and mass range of $30-300 \mathrm{~m} / \mathrm{z}$ in the full scan mode (scan time $0.4 \mathrm{~s}$ ). Data were collected using the GCMSsolution software ver. 2 (Shimadzu). The tentative identification of aromatic compounds was performed based on mass spectra and confirmed by linear retention index. The mass spectrum of each peak was compared to data provided in the National Institute of Standards and Technology mass spectral library (NIST 05). The peak was correctly identified when the similarity of the spectra was at least $80 \%$. Chromatographic retention data for $\mathrm{C}_{7}-\mathrm{C}_{30}$ $n$-alkanes (Supelco) were used to calculate the RI of each compound. Experimental RI results were compared to the retention indices found in the bibliography for similar GC columns. Data for the volatile compounds were calculated by relating their peak areas to the peak area of the internal standard. The contents of volatiles in wines were expressed as $\mu \mathrm{g} / \mathrm{L}$.

\section{Fourier transform infrared spectroscopy}

FTIR spectra were measured on a 670 -IR spectrometer from Agilent. FTIR measurements were made five times for each wine. An attenuated total reflection (ATR) trough in the form of a horizontal ATR (HATR) Ge crystal with an appropriate geometry (i.e., truncated at $45^{\circ}$ ) was used in the measurements. This was to ensure a 20 -fold internal reflection of the absorbed beam. Additionally, background correction was applied and 24 scans were recorded at the recording of each spectrum. Then, the program averaged the spectra obtained, before and after each measurement the crystal was thoroughly purified using ultra-pure and clear solvents from Sigma-Aldrich. The apparatus was continuously purged with argon before $(1 \mathrm{~h})$ and during the entire spectral measurement. FTIR spectra were recorded with a resolution of $1 \mathrm{~cm}^{-1}$ and measured in the range of 4000 to $400 \mathrm{~cm}^{-1}$. In the end, the spectra were analyzed with Grams/AI 8.0 software (Thermo Fisher Scientific, Waltham, MA, USA). All samples were measured at room temperature.

\section{Statistical analysis}

The effects of grape variety, yeast, MLF, and yeast $\times$ MLF interaction on the content of volatile compounds in wines were tested using one-way and two-way analysis of variance
(ANOVA). One- and two-way ANOVA was performed with the Statistica 13.3 software (Statsoft, Krakow, Poland).

Next, hierarchical cluster analysis (HCA) with Euclidean distance was applied as an unsupervised classification technique in order to explore the FTIR data structure. The hierarchical cluster analysis and dendrogram were performed with Statistica 13.3 software. Next, principal component analysis (PCA) was applied to verify the similarities and differences among the wines. The PCA was based on the data array of the fingerprints of FTIR spectra of each considered wine, and carried out using OriginPro software (OriginLab, Northampton, MA, USA).

\section{RESULTS AND DISCUSSION}

Forty-six volatile compounds belonging to several groups alcohols (21 compounds), esters (12 compounds), acids (5 compounds), aldehydes (2 compounds), ketones (2 compounds), furan compounds (2 compounds), sulfur compounds (1 compound) and volatile phenols (1 compound) - were identified in red wines produced from Zweigelt and Rondo grape varieties (Table 1). Figure S1 and Figure S2 in the supplementary materials present the chromatograms of volatile compounds of Zweigelt and Rondo wines, respectively. Table 2 and Table 3 present the concentrations of volatile compounds of Zweigelt and Rondo wines, respectively, produced with different yeast and MLF combinations.

The dominating alcohols in the wines produced from the Zweigelt variety were: 3-methylbutan-1-ol, 2-methylpropan-1-ol, and hexan-1-ol (Table 2). The concentrations of 2-(2-ethoxyethoxy)-ethanol, decan-1-ol and (Z)-2-hexen-1-ol were the lowest. The major esters were ethyl 2-hydroxypropanoate (ethyl lactate), ethyl octanoate, and 3-methylbutyl acetate, while minor ones were: 2-phenylethyl acetate, hexyl acetate, and methyl octanoate. Among the acids, acetic acid and hexanoic acid were found at the highest concentrations. Propanoic acid was detected only in Z2 LAB-Z5 LAB wines. Benzaldehyde was the most abundant compound in the two detected aldehydes, 4-methyl-3-penten-2-one was the most abundant compound in the two detected ketones, dihydrofuran-2(3H)-one was the most abundant in the two detected furan compounds.

The most abundant alcohols in wines produced from the Rondo variety were: 3-methylbutan-1-ol, 2-methylpropan-1-ol, and 2-phenylethanol (Table 3). Rondo wines had the highest concentration of 3-methylbutan-1-ol, similarly to our previous works [Stój et al., 2017a,b] and to the work of Liu et al. [2017]. Contrary to our findings, Ruocco et al. [2019] reported the highest content of 2,3-butanediol among all alcohols. The minor alcohols of Rondo wines were: 3-ethyl-4-methylpentan-1-ol, phenylmethanol, and decan-1-ol (Table 3). The dominating esters were: ethyl 2-hydroxypropanoate, 3-methylbutyl acetate, and ethyl octanoate, and it is in agreement with our previous works [Stój et al., 2017a,b]. According to Liu et al. [2017], ethyl octanoate and ethyl acetate were the main esters in wines produced from Rondo variety. In turn, Ruocco et al. [2019] stated that ethyl 2-hydroxypropanoate and ethyl acetate were the dominating esters in Rondo wines. The dissimilarities between 
TABLE 1. Volatile compounds identified in red wines.

\begin{tabular}{|c|c|c|c|c|c|c|}
\hline Peak no. & Compound & Similarity (\%) & $\mathrm{RT}$ (min) & RI exp. & RI lit. & References \\
\hline \multicolumn{7}{|c|}{ Alcohol } \\
\hline 2 & Propanol-1-ol & 95 & 13.50 & 1051 & 1044 & Mendes et al. [2012] \\
\hline 4 & 2-Methylpropan-1-ol & 97 & 16.31 & 1122 & 1100 & Mendes et al. [2012] \\
\hline 7 & Butan-1-ol & 96 & 18.73 & 1169 & 1173 & Welke et al. [2012] \\
\hline 8 & 3-Methylbutan-1-ol & 98 & 21.79 & 1228 & 1210 & Mendes et al. [2012] \\
\hline 9 & Pentan-1-ol & 94 & 23.46 & 1261 & 1259 & Mallouchos et al. [2007] \\
\hline 12 & 4-Methylpentan-1-ol & 95 & 26.35 & 1318 & 1309 & Duarte et al. [2010] \\
\hline 13 & 3-Methylpentan-1-ol & 97 & 26.94 & 1329 & 1322 & Duarte et al. [2010] \\
\hline 16 & Hexan-1-ol & 98 & 28.20 & 1353 & 1361 & Mallouchos et al. [2007] \\
\hline 17 & 3-Ethoxypropan-1-ol & 93 & 29.44 & 1376 & 1371 & Welke et al. [2012] \\
\hline 19 & (Z)-2-Hexen-1-ol & 87 & 31.73 & 1417 & 1397 & Welke et al. [2012] \\
\hline 21 & Octen-3-ol & 95 & 33.94 & 1453 & 1451 & Song et al. [2014] \\
\hline 22 & Heptan-1-ol & 97 & 34.24 & 1458 & 1470 & Welke et al. [2012] \\
\hline 24 & 2-Ethylhexan-1-ol & 98 & 36.19 & 1490 & 1486 & Duarte et al. [2010] \\
\hline 25 & 3-Ethyl-4-methylpentan-1-ol & 92 & 37.14 & 1508 & 1509 & Welke et al. [2012] \\
\hline 27 & Butane-2,3-diol & 98 & 38.61 & 1543 & 1563 & Welke et al. [2012] \\
\hline 29 & Octan-1-ol & 98 & 39.32 & 1559 & 1567 & Mallouchos et al. [2007] \\
\hline 32 & Propane-1,2-diol & 90 & 40.75 & 1592 & 1591 & Welke et al. [2012] \\
\hline 33 & 2-(2-Ethoxyethoxy)-ethanol & 89 & 41.67 & 1618 & 1622 & Welke et al. [2012] \\
\hline 40 & Decan-1-ol & 95 & 46.13 & 1760 & 1778 & Welke et al. [2012] \\
\hline 43 & Phenylmethanol & 84 & 49.27 & 1881 & 1869 & Duarte et al. [2010] \\
\hline \multirow[t]{2}{*}{44} & 2-Phenylethanol & 97 & 50.10 & 1916 & 1919 & Mallouchos et al. [2007] \\
\hline & Ester & & & & & \\
\hline 1 & Ethyl butanoate & 97 & 13.05 & 1037 & 1034 & Mallouchos et al. [2007] \\
\hline 3 & Ethyl 3-methylbutanoate & 94 & 14.43 & 1078 & 1066 & Duarte et al. [2010] \\
\hline 5 & 3-Methylbutyl acetate & 98 & 16.88 & 1133 & 1125 & Duarte et al. [2010] \\
\hline 10 & Hexyl acetate & 90 & 24.16 & 1275 & 1272 & Mallouchos et al. [2007] \\
\hline 14 & Ethyl heptanoate & 91 & 27.38 & 1337 & 1349 & Welke et al. [2012] \\
\hline 15 & Ethyl 2-hydroxypropanoate & 98 & 27.87 & 1346 & 1339 & Welke et al. [2012] \\
\hline 18 & Methyl octanoate & 85 & 30.01 & 1387 & 1381 & Welke et al. [2012] \\
\hline 20 & Ethyl octanoate & 97 & 32.85 & 1435 & 1429 & Welke et al. [2012] \\
\hline 30 & 3-Methylbutyl 2-hydroxypropanoate & 97 & 39.85 & 1572 & 1568 & Mendes et al. [2012] \\
\hline 36 & Ethyl decanoate & 88 & 42.38 & 1639 & 1643 & Welke et al. [2012] \\
\hline 38 & Diethyl butanedioate & 96 & 43.66 & 1677 & 1672 & Duarte et al. [2010] \\
\hline \multirow[t]{2}{*}{41} & 2-Phenylethyl acetate & 90 & 47.73 & 1819 & 1810 & Duarte et al. [2010] \\
\hline & Acid & & & & & \\
\hline 23 & Acetic acid & 98 & 34.59 & 1464 & 1457 & Welke et al. [2012] \\
\hline 28 & Propanoic acid & 86 & 39.02 & 1552 & 1536 & Welke et al. [2012] \\
\hline 31 & 2-Methylpropanoic acid & 97 & 40.11 & 1578 & 1573 & Mallouchos et al. [2007] \\
\hline
\end{tabular}


TABLE 1 continued

\begin{tabular}{|c|c|c|c|c|c|c|}
\hline Peak no. & Compound & Similarity $(\%)$ & $\mathrm{RT}$ (min) & RI exp. & RI lit. & References \\
\hline 42 & Hexanoic acid & 96 & 48.53 & 1851 & 1851 & Mallouchos et al. [2007] \\
\hline \multirow[t]{2}{*}{45} & Octanoic Acid & 95 & 53.38 & 2065 & 2067 & Mallouchos et al. [2007] \\
\hline & Aldehyde & & & & & \\
\hline 26 & Benzaldehyde & 91 & 37.90 & 1526 & 1522 & Mallouchos et al. [2007] \\
\hline \multirow[t]{2}{*}{37} & 4-Methylbenzaldehyde & 92 & 42.78 & 1651 & 1638 & Duarte et al. [2010] \\
\hline & Ketone & & & & & \\
\hline 6 & 4-Methyl-3-penten-2-one & 98 & 17.56 & 1146 & 1139 & Jørgensen et al. [2000] \\
\hline \multirow[t]{2}{*}{11} & 3-Hydroxybutan-2-one & 90 & 25.39 & 1299 & 1289 & Mallouchos et al. [2007] \\
\hline & Furan compound & & & & & \\
\hline 34 & Ethyl 2-furoate & 88 & 41.93 & 1625 & 1627 & Welke et al. [2012] \\
\hline \multirow[t]{2}{*}{35} & Dihydrofuran-2(3H)-one & 93 & 42.22 & 1634 & 1627 & Mallouchos et al. [2007] \\
\hline & Sulphur compound & & & & & \\
\hline \multirow[t]{2}{*}{39} & 3-(Methylsulfanyl)propan-1-ol & 96 & 44.95 & 1718 & 1715 & Duarte et al. [2010] \\
\hline & Volatile phenol & & & & & \\
\hline 46 & 3,5-Di-tert-butylphenol & 86 & 58.08 & 2305 & 2310 & Shimoda et al. [1995] \\
\hline
\end{tabular}

RT - retention time; RI exp. -retention index experimentally determined; RI lit. -retention index reported in the literature for a CP-Wax columns or equivalent stationary phase. Peak no. correspond to those in the chromatograms in Figures S1 and S2 in the supplementary materials.

concentrations of volatile compounds among studies may have been due to differences in geographical origins of grapes, winemaking, and methods of determination of volatile compounds. Acetic acid was present at the highest concentration among the acids of Rondo wines (Table 3), while propanoic acid was not detected. Similarly, other authors reported that acetic acid was the major acid of wines produced using Rondo grapes [Liu et al., 2017; Ruocco et al., 2019] and we also stated this in our previous publications [Stój et al., 2017a,b]. Major volatile compounds within the other classes were: benzaldehyde, 4-methyl-3-penten-2-one, and dihydrofuran-2(3H)-one (Table 3 ).

A one-way ANOVA was used to study the grape variety/ yeast/MLF effect on volatile compound profile of wines. Results of this analysis revealed a significant effect of grape variety, yeast, and MLF on the concentrations of 32, 7, and 10 volatile compounds, respectively, belonging to all groups of compounds (Table 4). The grape variety was the factor with a significant impact on the highest number of identified volatile compounds. The two-way ANOVA confirmed the effects of yeast and MLF on volatile compounds in wines (Table 4). The yeast and MLF factors had a significant impact on a greater number of volatile compounds compared to the yeast $\times$ MLF interaction, which affected concentrations of only three compounds: 2-phenylethanol, octanoic acid and 3-(methylsulfanyl)propan-1-ol.

In our study, the grape variety effect was significant for most alcohols, including: propanol-1-ol, butan-1-ol, 3-methylbutan-1-ol, pentan-1-ol, 4-methylpentan-1-ol, 3-methylpentan-1-ol, hexan-1-ol, 3-ethoxypropan-1-ol, (Z)-2-hexen-1-ol, octen-3-ol, heptan-1-ol, 2-ethylhexan-1-ol, 3-ethyl-4-methylpentan-1-ol, octan-1-ol, decan-1-ol, phenylmethanol, and 2-phenylethanol (Table 4), while Liu et al. [2017] reported that the concentration of all determined alcohols was affected by the variety. Higher alcohols are strictly related to yeast metabolism [Blanco et al., 2014; Callejon et al., 2010]. They could be synthesized by yeast through two mechanisms: the anabolic pathway from glucose or the catabolic pathway from their corresponding amino acids [Liu et al., 2017; Stój et al., 2017a]. Yeast species and strains (and other factors such as $\mathrm{pH}$, grape juice composition and fermentation temperature) influence the formation of higher alcohols during fermentation [Liu et al., 2017]. In our study, concentrations of 3-ethoxypropan-1-ol, (Z)-2-hexen-1-ol, phenylmethanol, and 2-phenylethanol were significantly affected by yeast strain used in winemaking. Both Blanco et al. [2014] and Tufariello et al. [2014] found no differences in phenylmethanol concentration, whereas Callejon et al. [2010] and Tufariello et al. [2014] found no differences in 2-phenylethanol concentration between red wines produced using different yeast strains. Similarly to our study, Callejon et al. [2010] reported a correlation of phenylmethanol concentration with a yeast strain, and Blanco et al. [2014] reported a correlation of 2-phenylethanol content with a yeast strain. The yeast strains used in this study did not influence the concentrations of other higher alcohols, such as propanol-1-ol, 2-methylpropan-1-ol, butan-1-ol, 3-methylbutan-1-ol, hexan-1-ol, heptan-1-ol (Table 4). Blanco et al. [2014], Callejon et al. [2010], and Tufariello et al. [2014] observed the effect of yeast strain or no effect on the concentrations of higher alcohols. Finally, the MLF 


\begin{tabular}{|c|c|c|c|c|c|c|c|c|c|c|c|c|c|c|c|c|c|c|c|c|c|c|c|c|c|c|c|}
\hline $\begin{array}{l}0 \\
+1 \\
+1 \\
\bar{E} \\
\sum\end{array}$ & & 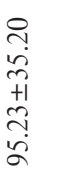 & 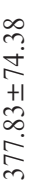 & 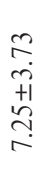 & 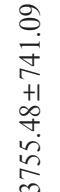 & $\begin{array}{l}\infty \\
0 \\
0 \\
+1 \\
0 \\
0 \\
i\end{array}$ & 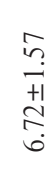 & $\begin{array}{l}\stackrel{\alpha}{\alpha} \\
\stackrel{+}{+1} \\
\infty \\
\infty \\
\infty\end{array}$ & 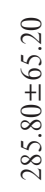 & 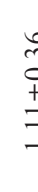 & 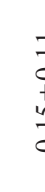 & & 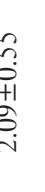 & 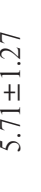 & $\begin{array}{l}\hat{\imath} \\
0 \\
+1 \\
n \\
\hat{n} \\
\hat{\sim}\end{array}$ & 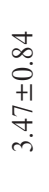 & $\begin{array}{l}m \\
\infty \\
\infty \\
+1 \\
+1 \\
\dot{\sigma}\end{array}$ & 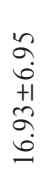 & $\begin{array}{l}\stackrel{m}{=} \\
\stackrel{+}{+} \\
\stackrel{+}{-}\end{array}$ & 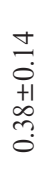 & $\begin{array}{l}8 \\
0 \\
0 \\
+1 \\
0 \\
0 \\
0\end{array}$ & $\begin{array}{l}\infty \\
\stackrel{\infty}{0} \\
+1 \\
\stackrel{1}{0} \\
0\end{array}$ & $\begin{array}{l}\infty \\
\stackrel{+}{+} \\
\overrightarrow{+} \\
+1 \\
\dot{d} \\
\dot{d} \\
\underline{d}\end{array}$ & & 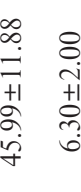 & & \\
\hline 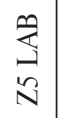 & & $\underset{\Theta}{\stackrel{\Delta}{\Theta}}$ & $\begin{array}{l}\frac{\infty}{\vec{r}} \\
\stackrel{f}{f}\end{array}$ & $\stackrel{n}{n}$ & $\begin{array}{l}R \\
b \\
i n \\
m\end{array}$ & $\stackrel{\circ}{\stackrel{i}{i}}$ & $\begin{array}{l}\text { f } \\
\text { in }\end{array}$ & ف & $\begin{array}{l}\dot{\alpha} \\
\dot{a}\end{array}$ & & 7 & 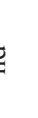 & : & & $\stackrel{\text { f. }}{=}$ & ત્ & 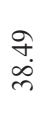 & $\begin{array}{l}\widetilde{\sigma} \\
\stackrel{+}{ \pm}\end{array}$ & లి & 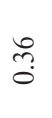 & $\vec{Z}$ & $\stackrel{ \pm}{\circ}$ & $\begin{array}{l}\tilde{\infty} \\
\dot{d} \\
\dot{g}\end{array}$ & & 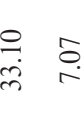 & & \\
\hline $\begin{array}{l}\underset{J}{J} \\
\underset{N}{J}\end{array}$ & & $\frac{\overrightarrow{\vec{b}}}{\vec{n}}$ & $\begin{array}{l}\stackrel{0}{\circ} \\
\stackrel{\leftrightarrow}{\Im}\end{array}$ & $\frac{0}{6}$ & $\begin{array}{l}\vec{\sigma} \\
\dot{\vec{a}} \\
\ddot{n}\end{array}$ & $\stackrel{0}{i}$ & $\stackrel{\Re}{q}$ & 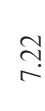 & $\begin{array}{l}\stackrel{8}{0} \\
\infty \\
\stackrel{\Delta}{d}\end{array}$ & 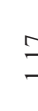 & ઈิ & & 过 & 6 & $\underset{\infty}{\stackrel{\Gamma}{\infty}}$ & $\stackrel{\infty}{+\infty}$ & $\begin{array}{l}m \\
\hat{\sigma} \\
\hat{\sigma}\end{array}$ & $\begin{array}{l}\tilde{a} \\
\dot{I}\end{array}$ & $\stackrel{\sigma}{-}$ & F. & $\stackrel{m}{0}$ & $\stackrel{\infty}{\circ}$ & $\begin{array}{l}\stackrel{n}{\stackrel{d}{c}} \\
\stackrel{\infty}{a}\end{array}$ & & $\begin{array}{ll}\hat{n} & \bar{n} \\
\bar{m} & \bar{m}\end{array}$ & s & \\
\hline 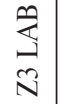 & & 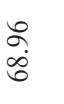 & 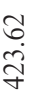 & 疋 & 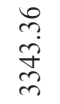 & $\stackrel{\overbrace{}}{i}$ & $\stackrel{\bar{\infty}}{\sim}$ & 항 & $\stackrel{\substack{a \\
\dot{d}}}{\sim}$ & $f$ & 9 & & $\overrightarrow{\mathrm{N}}$ & 6 & 守 & $\stackrel{f}{\dot{q}}$ & $\begin{array}{l}\stackrel{\infty}{ } \\
\dot{y}\end{array}$ & $\begin{array}{l}\tilde{\infty} \\
\ddot{n}\end{array}$ & $\exists$ & $\stackrel{n}{o}$ & $\stackrel{\circ}{\circ}$ & $\stackrel{\circ}{0}$ & $\begin{array}{l}\stackrel{0}{\infty} \\
\stackrel{\infty}{=}\end{array}$ & & $\vec{f}$ & is & \\
\hline $\begin{array}{l}\underset{Z}{J} \\
\text { N }\end{array}$ & & 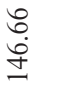 & 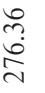 & 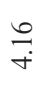 & $\begin{array}{l}\tilde{\sigma} \\
\stackrel{\vec{n}}{n}\end{array}$ & i̊ & $\stackrel{t}{\sigma}$ & $\stackrel{2}{\sigma}$ & $\begin{array}{l}\infty \\
\stackrel{\infty}{0} \\
\stackrel{\sim}{0}\end{array}$ & " & z) & & $\tilde{\hat{v}}$ & in & $\begin{array}{l}\text { to } \\
\infty \\
\infty \\
\infty\end{array}$ & $\vec{n}$ & $\underset{\dot{m}}{\stackrel{ \pm}{m}}$ & $\stackrel{\cong}{=}$ & $\bar{n}$ & $\overline{\widetilde{\sigma}}$ & $\stackrel{\overbrace{}}{8}$ & $\stackrel{\infty}{\circ}$ & $\begin{array}{l} \pm \\
\infty \\
心 \\
\stackrel{d}{a}\end{array}$ & & 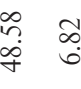 & $\begin{array}{l}\infty \\
\sigma \\
\sigma\end{array}$ & 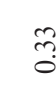 \\
\hline $\begin{array}{l}\stackrel{m}{3} \\
\vec{N}\end{array}$ & & $\stackrel{\overline{\mathrm{c}}}{\stackrel{\Xi}{\varrho}}$ & $\begin{array}{l}\vec{\infty} \\
\vec{j} \\
\vec{m}\end{array}$ & $\stackrel{\overbrace{}}{\stackrel{\overbrace{}}{g}}$ & 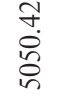 & $\stackrel{\infty}{\stackrel{\infty}{\infty}}$ & $\begin{array}{l}\infty \\
0 \\
\infty\end{array}$ & $\stackrel{\text { ? }}{=}$ & $\underset{\substack{\infty \\
\infty}}{\infty}$ & is & 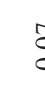 & & $\mathbb{\infty}_{i}^{\infty}$ & है & $\stackrel{\overbrace{}}{\tilde{f}}$ & ț & $\begin{array}{l}\text { f. } \\
\stackrel{8}{\infty}\end{array}$ & $\begin{array}{l}\infty \\
\stackrel{\infty}{d} \\
\dot{\sim}\end{array}$ & $\underset{m}{ \pm}$ & $\stackrel{0}{\circ}$ & 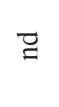 & $\stackrel{+}{n}$ & 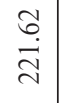 & & 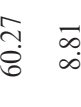 & כ & . \\
\hline $\begin{array}{l}0 \\
+1 \\
+1 \\
\bar{\Xi} \\
\sum_{\Sigma}^{ \pm}\end{array}$ & & 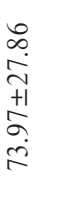 & 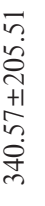 & $\begin{array}{l}8 \\
0 \\
\dot{0} \\
+1 \\
+ \\
0 \\
0\end{array}$ & 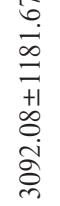 & 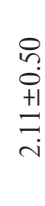 & $\begin{array}{l}\vec{\sigma} \\
+1 \\
+1 \\
0 \\
\dot{n}\end{array}$ & $\begin{array}{l}\stackrel{8}{n} \\
\stackrel{+1}{+} \\
\stackrel{+}{r}\end{array}$ & 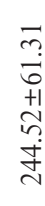 & $\begin{array}{ll}5 \\
0 \\
0 \\
0 \\
0 \\
0\end{array}$ & 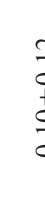 & 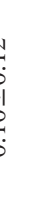 & 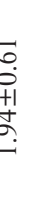 & $\frac{\dot{i}}{+1}$ & 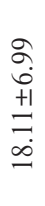 & $\begin{array}{l}\text { aे } \\
\dot{0} \\
+1 \\
\stackrel{1}{0} \\
\dot{m}\end{array}$ & 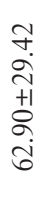 & 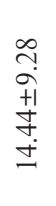 & $\begin{array}{l}\widetilde{d} \\
i \\
+1 \\
\stackrel{0}{0} \\
i\end{array}$ & $\begin{array}{l}\stackrel{\Upsilon}{1} \\
0 \\
+1 \\
0 \\
0 \\
0\end{array}$ & $\begin{array}{l}\frac{a}{0} \\
0 \\
+1 \\
\stackrel{0}{0}\end{array}$ & 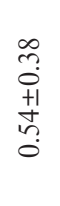 & $\begin{array}{l}n \\
\infty \\
\infty \\
o \\
+1 \\
0 \\
o \\
c \\
=\end{array}$ & & 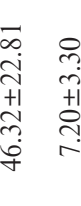 & fే & 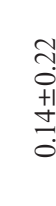 \\
\hline 3 & & $\begin{array}{l}\infty \\
\stackrel{\alpha}{\alpha}\end{array}$ & ל̂. & 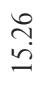 & $\stackrel{\text { בे }}{\overrightarrow{\bar{B}}}$ & $\begin{array}{l}\infty \\
\infty \\
i\end{array}$ & $\underset{\infty}{\infty}$ & $\stackrel{\circ}{\circ}$ & ले & in & 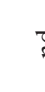 & to & $\hat{i}^{\infty}$ & 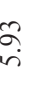 & $\begin{array}{l}\text { o } \\
\text { ¿े }\end{array}$ & $\stackrel{\infty}{\stackrel{+}{+}}$ & $\begin{array}{l}\bar{\sigma} \\
\infty \\
\stackrel{0}{0}\end{array}$ & $\stackrel{\text { के }}{\overrightarrow{0}}$ & $\begin{array}{l}\widetilde{\sigma} \\
i \\
i\end{array}$ & $\stackrel{8}{\circ}$ & $\stackrel{\infty}{\stackrel{0}{0}}$ & $\stackrel{\infty}{\stackrel{0}{0}}$ & $\begin{array}{l}\vec{b} \\
\stackrel{1}{d} \\
\text { d. }\end{array}$ & & 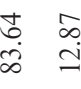 & $\bar{z}$ & $\Xi$ \\
\hline$\stackrel{+}{N}$ & & $\begin{array}{l}\underset{+}{+} \\
\dot{+}\end{array}$ & 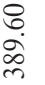 & $\bar{g}$ & 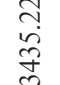 & $\stackrel{\vec{i}}{\mathrm{i}}$ & $\stackrel{\infty}{\circ}$ & $\stackrel{f}{\stackrel{f}{r}}$ & 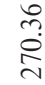 & $\alpha$ & o & $\dot{b}$ & $\infty_{i}^{\infty}$ & $\begin{array}{l}\infty \\
\infty \\
\dot{f} \\
+\end{array}$ & $\begin{array}{l}\text { त̂ } \\
\text { ڤn }\end{array}$ & बे & $\begin{array}{l}\stackrel{\infty}{\infty} \\
\underset{m}{m}\end{array}$ & $\stackrel{\sigma}{\alpha}$ & $\stackrel{\infty}{\infty}$ & ণิ & $\stackrel{\circ}{\circ}$ & $\stackrel{0}{\stackrel{0}{0}}$ & 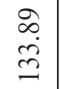 & & لَّ & $\begin{array}{l}\infty \\
\neq \\
j\end{array}$ & ฮี \\
\hline$\hat{N}$ & & $\frac{\infty}{\dot{d}}$ & مَ & $\stackrel{\infty}{\stackrel{\sim}{+}}$ & $\begin{array}{l}\infty \\
\infty \\
\infty \\
\infty \\
\vec{N}\end{array}$ & $\underset{\text { i }}{\stackrel{8}{ }}$ & तु่ & $\stackrel{\circ}{\circ}$ & 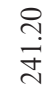 & 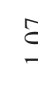 & ( & ? & S & 3 & $\begin{array}{l}\text { ते } \\
\text { ते }\end{array}$ & $\underset{\mathrm{d}}{\mathrm{r}}$ & $\underset{m}{\stackrel{m}{m}}$ & $\begin{array}{l}\stackrel{2}{\circ} \\
\text { అે }\end{array}$ & $\stackrel{\mathscr{O}}{\mathscr{O}}$ & $\stackrel{\widetilde{o}}{3}$ & $\stackrel{\overbrace{}}{0}$ & $\stackrel{7}{0}$ & 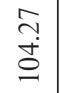 & & 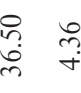 & $\begin{array}{l}\text { in } \\
\text { ñ }\end{array}$ & ถึ \\
\hline $\mathbb{N}$ & & $\begin{array}{l}\vec{\sigma} \\
\text { gे }\end{array}$ & פ্ & $\underset{\infty}{\infty}$ & $\begin{array}{l}\text { बे } \\
\overrightarrow{0} \\
\text { ते }\end{array}$ & $\underline{\sqrt[n]{-}}$ & ָึ & $\begin{array}{l}\infty \\
\infty \\
i\end{array}$ & 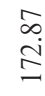 & $\approx$ & 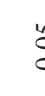 & 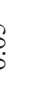 & $\stackrel{\circ}{\circ}$ & $\infty$ & $\begin{array}{l}\stackrel{\infty}{\infty} \\
= \\
=\end{array}$ & $\stackrel{\overbrace{}}{i}$ & $\begin{array}{l}\overrightarrow{0} \\
\dot{b}\end{array}$ & oे & $\begin{array}{l}n \\
n \\
n\end{array}$ & $\stackrel{0}{0}$ & $\vec{g}$ & $\stackrel{8}{\circ}$ & $\begin{array}{l}\infty \\
\stackrel{\vdots}{\sigma} \\
\tilde{g}\end{array}$ & & 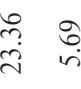 & in & $\Xi$ \\
\hline $\bar{N}$ & & $\begin{array}{l}\vec{F} \\
\stackrel{\leftrightarrow}{\circ}\end{array}$ & 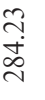 & ?f & 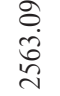 & $\stackrel{\infty}{\stackrel{\infty}{-}}$ & 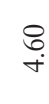 & శ్రి & 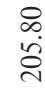 & & 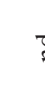 & 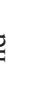 & 冓 & & $\stackrel{\stackrel{\circ}{\Xi}}{=}$ & $\stackrel{\vec{i}}{i}$ & $\begin{array}{l}\bar{\sigma} \\
\dot{0}\end{array}$ & $\stackrel{n}{\stackrel{n}{I}}$ & $\underset{i}{ \pm}$ & $\tilde{3}$ & $\stackrel{ \pm}{0}$ & $\stackrel{ \pm}{\Xi}$ & $\begin{array}{l}8 \\
\dot{0} \\
\dot{b} \\
-\end{array}$ & & 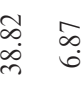 & है & $\Xi$ \\
\hline $\begin{array}{l}\text { 言 } \\
\text { : } \\
\text { : }\end{array}$ & $\begin{array}{l}\overline{0} \\
\text { o. } \\
\frac{0}{2}\end{array}$ & 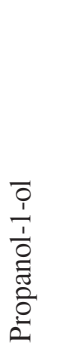 & 产 & 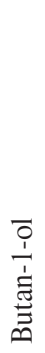 & 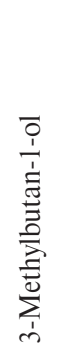 & 莖 & 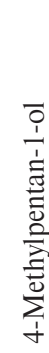 & 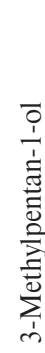 & 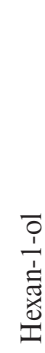 & 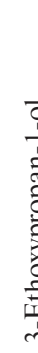 & $=$ & 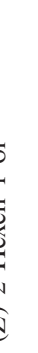 & 它 & $\stackrel{i}{I}$ & 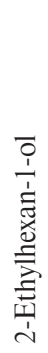 & 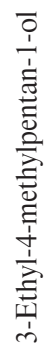 & 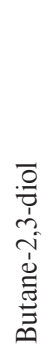 & $\begin{array}{l}\overline{0} \\
\frac{1}{1} \\
\text { 离 } \\
0\end{array}$ & 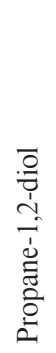 & 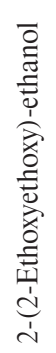 & 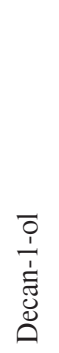 & 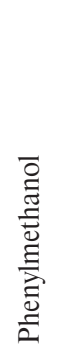 & 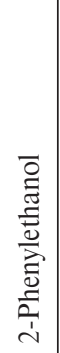 & 离 & 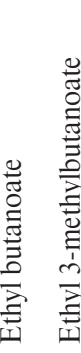 & $\sum_{i}^{\bar{a}}$ & $\overline{2}$ \\
\hline
\end{tabular}




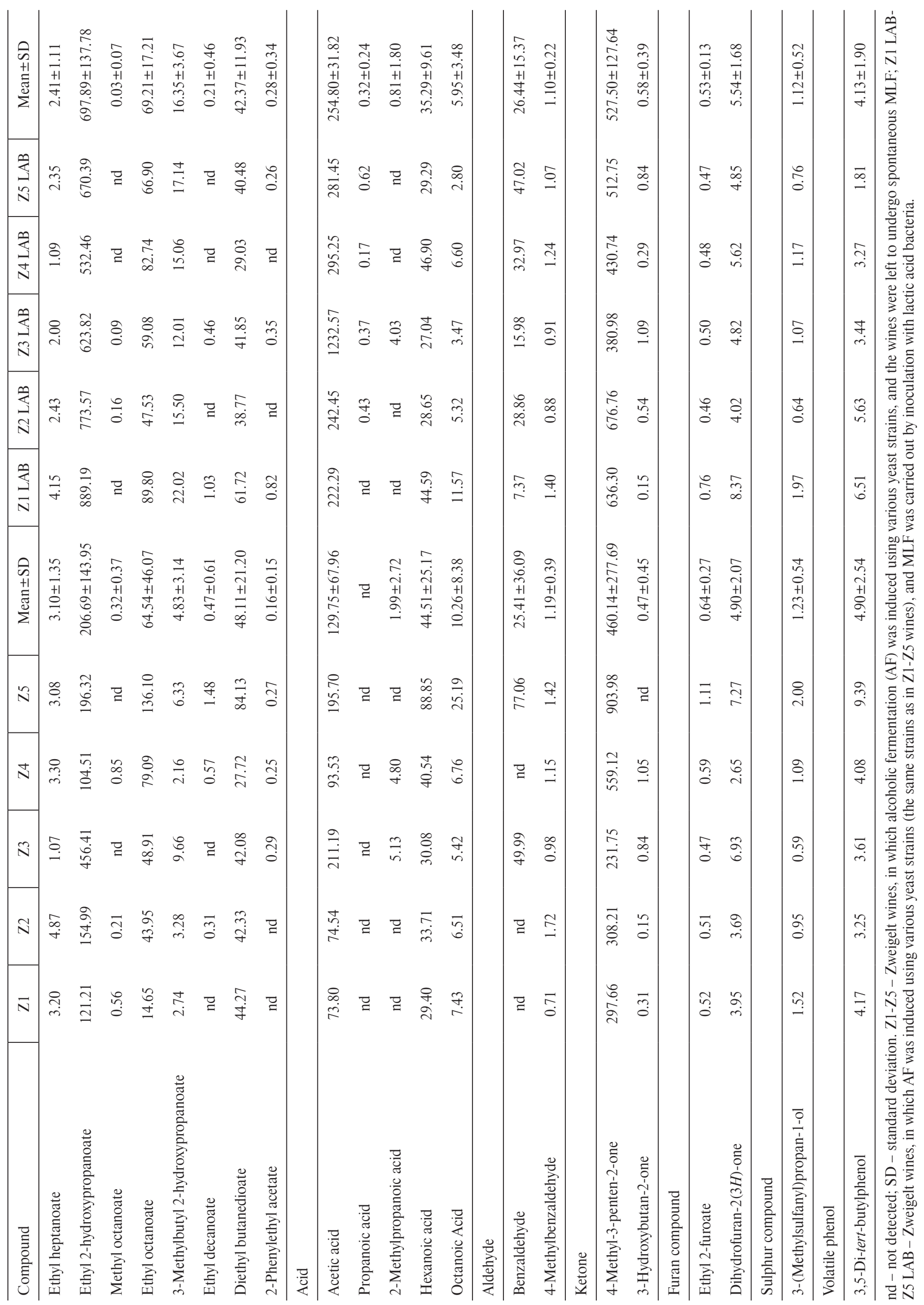




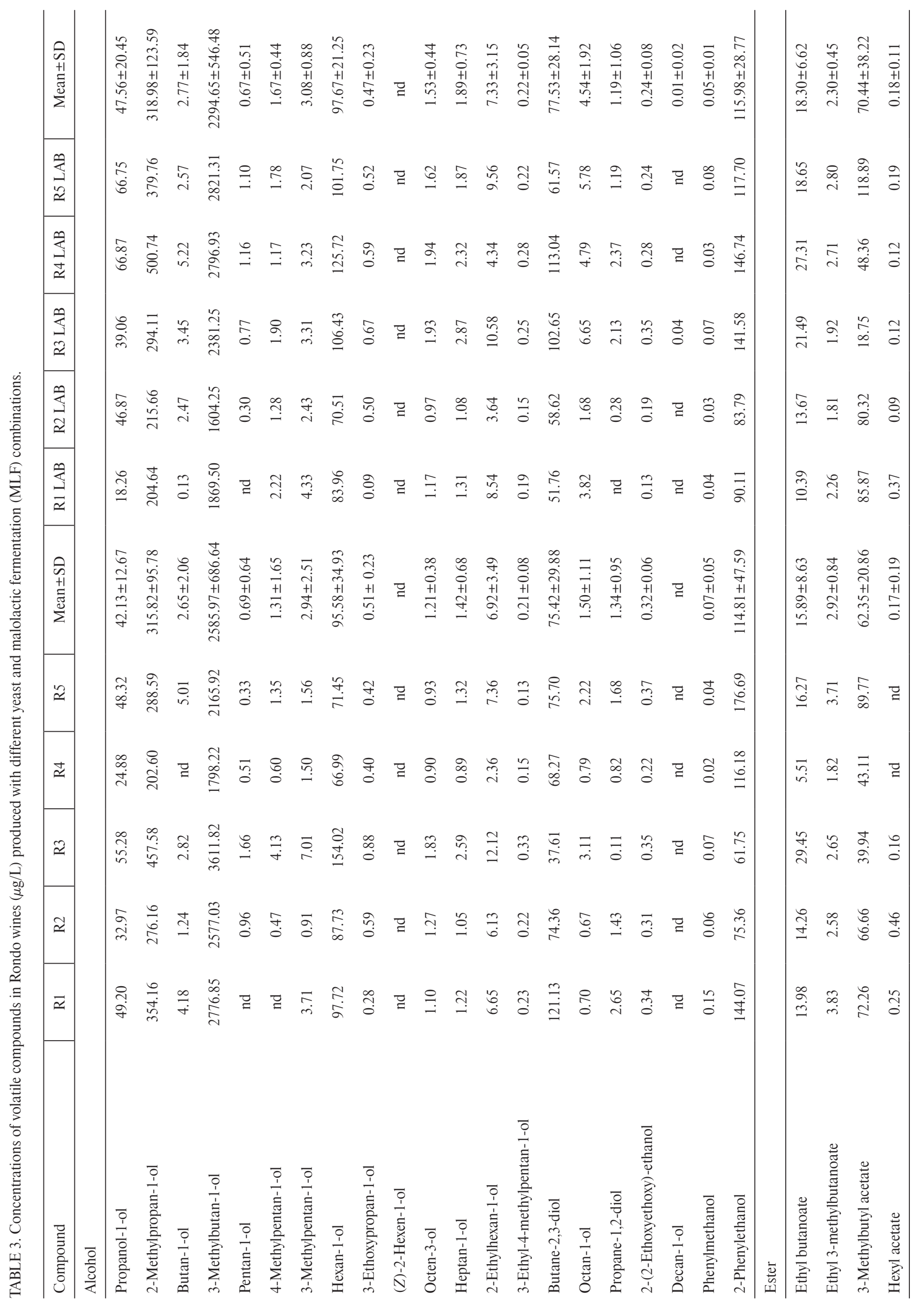




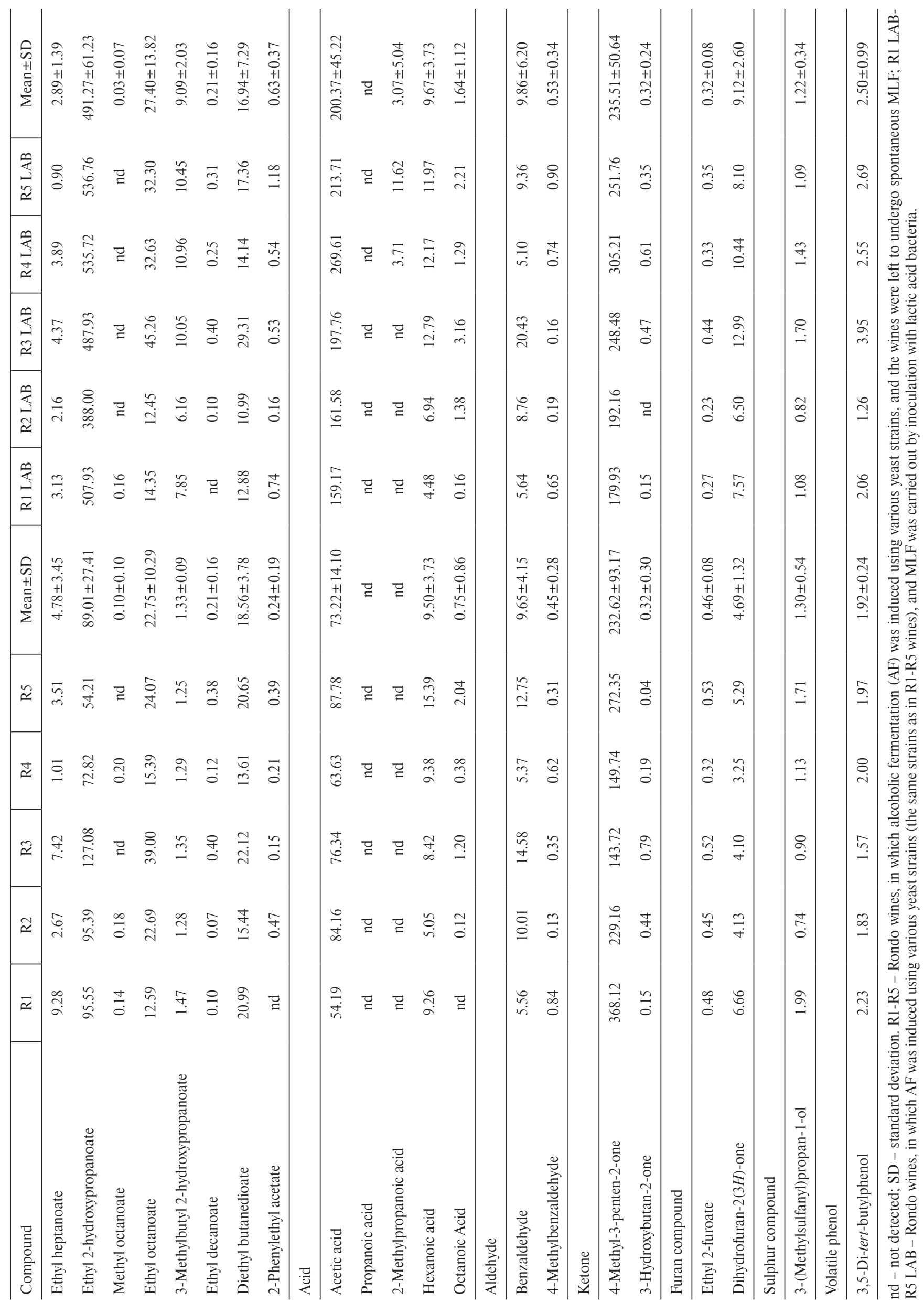


TABLE 4. Results of one-way analysis of variance (ANOVA) and two-way ANOVA of Rondo and Zweigelt wines produced with different yeast and malolactic fermentation (MLF) strategies.

\begin{tabular}{|c|c|c|c|c|c|c|}
\hline \multirow{2}{*}{ Compound } & \multicolumn{3}{|c|}{ One-way ANOVA } & \multicolumn{3}{|c|}{ Two-way ANOVA } \\
\hline & $\begin{array}{l}\text { Grape variety } \\
\text { effect }\end{array}$ & Yeast effect & MLF effect & Yeast effect & MLF effect & $\begin{array}{c}\text { Yeast } \times \text { MLF } \\
\text { effect }\end{array}$ \\
\hline \multicolumn{7}{|l|}{ Alcohol } \\
\hline Propanol-1-ol & $* * * *$ & NS & NS & NS & NS & NS \\
\hline 2-Methylpropan-1-ol & NS & NS & NS & NS & NS & NS \\
\hline Butan-1-ol & $* *$ & NS & NS & NS & NS & NS \\
\hline 3-Methylbutan-1-ol & $* *$ & NS & NS & NS & NS & NS \\
\hline Pentan-1-ol & $* * *$ & NS & NS & NS & NS & NS \\
\hline 4-Methylpentan-1-ol & $* * *$ & NS & NS & NS & NS & NS \\
\hline 3-Methylpentan-1-ol & $* * *$ & NS & NS & NS & NS & NS \\
\hline Hexan-1-ol & $* * *$ & NS & NS & NS & NS & NS \\
\hline 3-Ethoxypropan-1-ol & $* * *$ & $* * *$ & NS & $* * * *$ & NS & NS \\
\hline (Z)-2-Hexen-1-ol & $* * *$ & * & NS & * & NS & NS \\
\hline Octen-3-ol & * & NS & NS & NS & NS & NS \\
\hline Heptan-1-ol & $* * *$ & NS & NS & NS & NS & NS \\
\hline 2-Ethylhexan-1-ol & * & NS & NS & NS & NS & NS \\
\hline 3-Ethyl-4-methylpentan-1-ol & $* * *$ & NS & NS & NS & NS & NS \\
\hline Butane-2,3-diol & NS & NS & NS & NS & NS & NS \\
\hline Octan-1-ol & $* * *$ & NS & NS & NS & NS & NS \\
\hline Propane-1,2-diol & NS & NS & NS & NS & NS & NS \\
\hline 2-(2-Ethoxyethoxy)-ethanol & NS & NS & NS & NS & NS & NS \\
\hline Decan-1-ol & $*$ & NS & NS & NS & NS & NS \\
\hline Phenylmethanol & $* * *$ & $*$ & $*$ & $* *$ & * & NS \\
\hline 2-Phenylethanol & $* *$ & $* *$ & NS & $* *$ & NS & $* *$ \\
\hline \multicolumn{7}{|l|}{ Ester } \\
\hline Ethyl butanoate & $* * *$ & NS & NS & NS & NS & NS \\
\hline Ethyl 3-methylbutanoate & $* * *$ & NS & NS & NS & NS & NS \\
\hline 3-Methylbutyl acetate & NS & NS & $*$ & NS & $*$ & NS \\
\hline Hexyl acetate & NS & NS & NS & NS & NS & NS \\
\hline Ethyl heptanoate & NS & NS & $*$ & NS & $*$ & NS \\
\hline Ethyl 2-hydroxypropanoate & * & NS & $* * *$ & NS & $* * *$ & NS \\
\hline Methyl octanoate & NS & NS & * & NS & $*$ & NS \\
\hline Ethyl octanoate & $* *$ & NS & NS & NS & NS & NS \\
\hline 3-Methylbutyl 2-hydroxypropanoate & $* * *$ & NS & $* * *$ & NS & $* * *$ & NS \\
\hline Ethyl decanoate & NS & NS & NS & NS & NS & NS \\
\hline Diethyl butanedioate & $* * * *$ & NS & NS & NS & NS & NS \\
\hline 2-Phenylethyl acetate & NS & NS & NS & NS & NS & NS \\
\hline
\end{tabular}


TABLE 4 continued

\begin{tabular}{|c|c|c|c|c|c|c|}
\hline \multirow[b]{2}{*}{ Compound } & \multicolumn{3}{|c|}{ One-way ANOVA } & \multicolumn{3}{|c|}{ Two-way ANOVA } \\
\hline & $\begin{array}{c}\text { Grape variety } \\
\text { effect }\end{array}$ & Yeast effect & MLF effect & Yeast effect & MLF effect & $\begin{array}{c}\text { Yeast } \times \text { MLF } \\
\text { effect }\end{array}$ \\
\hline \multicolumn{7}{|l|}{ Acid } \\
\hline Acetic acid & $*$ & NS & $* * * *$ & NS & $* * *$ & NS \\
\hline Propanoic acid & $* *$ & NS & $* *$ & NS & $* *$ & NS \\
\hline 2-Methylpropanoic acid & NS & NS & NS & NS & NS & NS \\
\hline Hexanoic acid & **** & NS & NS & NS & NS & NS \\
\hline Octanoic acid & $* * *$ & NS & NS & NS & NS & $*$ \\
\hline \multicolumn{7}{|l|}{ Aldehyde } \\
\hline Benzaldehyde & $* *$ & **** & NS & $* * * *$ & NS & NS \\
\hline 4-Methylbenzaldehyde & $* * *$ & NS & NS & NS & NS & NS \\
\hline \multicolumn{7}{|l|}{ Ketone } \\
\hline 4-Methyl-3-penten-2-one & $* * *$ & NS & NS & NS & NS & NS \\
\hline 3-Hydroxybutan-2-one & NS & $* *$ & NS & $* * *$ & NS & NS \\
\hline \multicolumn{7}{|l|}{ Furan compound } \\
\hline Ethyl 2-furoate & $* * *$ & NS & $*$ & NS & $*$ & NS \\
\hline Dihydrofuran-2(3H)-one & NS & NS & $* *$ & NS & $* *$ & NS \\
\hline \multicolumn{7}{|l|}{ Sulphur compound } \\
\hline 3-(Methylsulfanyl)propan-1-ol & NS & $* *$ & NS & $* *$ & NS & $* *$ \\
\hline \multicolumn{7}{|l|}{ Volatile phenol } \\
\hline 3,5-Di-tert-butylphenol & $* *$ & NS & NS & NS & NS & NS \\
\hline
\end{tabular}

Statistical significance: *significant at $p<0.05,{ }^{* *}$ significant at $p<0.01,{ }^{* * *}$ significant at $p<0.001, \mathrm{NS}-$ not significant.

and yeast $\times$ MLF interaction influenced the concentrations of phenylmethanol and 2-phenylethanol, respectively (Table 4). Regarding yeast $\times$ MLF interaction, according to Gammacurta et al. [2017], 3-methylbutan-1-ol was the only higher alcohol not affected by yeast/LAB combination, while concentrations of other alcohols, such as propan-1-ol, 2-methylpropan-1-ol, and 2-methylbutan-1-ol, differed significantly depending on yeast/LAB combination.

Concentrations of half of the esters: ethyl butanoate, ethyl 3-methylbutanoate, ethyl 2-hydroxypropanoate, ethyl octanoate, 3-methylbutyl 2-hydroxypropanoate, and diethyl butanedioate differed significantly according to the grape variety (Table 4). However, Liu et al. [2017] found that grape variety significantly impacted concentrations of most esters. Ethyl esters are produced enzymatically by yeast from ethanolysis of acetyl-CoA formed during fatty acid synthesis or degradation [Liu et al., 2017; Stój et al., 2017a]. The concentrations of any esters were not significantly affected by yeast strain nor by yeast $\times$ MLF interaction (Table 4). On the contrary, Gammacurta et al. [2014] observed a significant effect of yeast strain on all esters in the red wines studied. Blanco et al. [2014] found that the concentrations of several esters (ethyl butanoate, hexyl acetate, 2-phenylethyl acetate, diethyl butanedioate) were independent of yeast strain, while other esters (3-methylbutyl acetate and ethyl octanoate) were yeast strain-dependent. Regarding yeast $\times$ MLF interaction, Gammacurta et al. [2017] reported that among 40 quantified esters, only seven were not affected by the yeast/LAB combination. Among seven esters, the concentrations of hexyl acetate and ethyl octanoate (also identified in our study) did not depend on the combination of microorganisms. MLF significantly influenced the concentrations of almost half of the esters, i.e. 3-methylbutyl acetate (isoamyl acetate), ethyl heptanoate, ethyl 2-hydroxypropanoate (ethyl lactate), methyl octanoate, and 3-methylbutyl 2-hydroxypropanoate (isoamyl lactate). Gammacurta et al. [2014] considered that the impact of LAB on esters was controversial because results differed between studies. Several reports showed changes in ester concentrations in wines after MLF with $O$. oeni due to its esterase activity [Brizuela et al., 2018; Diez-Ozaeta et al., 2021; Sumby et al., 2013]. However, Gammacurta et al. [2014] found that levels of esters were slightly affected by the LAB. In our study, the ethyl lactate concentration was higher in the wines subjected to induced MLF than in those subjected to spontaneous MLF. Ethyl lactate is one 
of the most characteristic aromatic compounds produced during MLF and a marker of LAB activity. Its content increased following MLF [Abrahamse \& Bartowsky, 2012; Costello et al., 2012; Lasik-Kurdyś et al., 2018].

We observed a grape variety effect on most acids, i.e. acetic acid, propanoic acid, hexanoic acid, and octanoic acid, whereas Liu et al. [2017] found significant differences for almost half of the acids. Fatty acids could be produced via anabolic pathways by yeast or during $\beta$-oxidation of long-chain fatty acids [Stój et al., 2017a]. The concentrations of any acids did not vary depending on yeast strain (Table 4). According to Blanco et al. [2014], only the hexanoic acid content of red wines depended on the yeast strain. MLF significantly modulated half of the acid (acetic acid and propanoic acid) contents in our study (Table 4). The concentration of acetic acid was higher in the wines subjected to induced MLF than in those subjected to spontaneous MLF (Table 2 and Table 3). The increase in acetic acid content is due to the metabolism of citric acid [Abrahamse \& Bartowsky, 2012; Styger et al., 2011]. The octanoic acid concentration was significantly impacted by yeast $\times$ MLF interaction (Table 4), which agreed with the results obtained by Gammacurta et al. [2017]. Concentrations of 2-methylpropanoic acid and hexanoic acid were not significantly modulated by yeast/LAB combinations, while Gammacurta et al. [2017] reported significant differences in the contents of these acids between microorganism combinations.

Regarding the two quantified aldehydes, concentrations of benzaldehyde and 4-methylbenzaldehyde differed significantly between grape varieties (Table 4). Yeast strain significantly impacted the concentration of benzaldehyde, similarly to the work of Tufariello et al. [2014] and contrary to the work of Blanco et al. [2014]. Neither MLF nor yeast $\times$ MLF interaction affected aldehyde concentrations.

The concentration of 4-methyl-3-penten-2-one differed significantly between wines produced from different grape varieties (Table 4). Grape variety did not affect 3-hydroxybutan-2-one (acetoin) concentration, contrary to the report of Liu et al. [2017]. The concentration of 3-hydroxybutan-2-one was significantly affected by yeast strain, which agreed with results obtained by Blanco et al. [2014]. The concentrations of the two identified ketones did not differ significantly upon the influence of either MLF or yeast $\times$ MLF interaction (Table 4). Considering the interactions, Gammacurta et al. [2017] observed no significant differences in the concentration of 3-hydroxybutan-2-one. Acetoin is produced from the metabolism of citric acid by lactic acid bacteria [Malherbe et al., 2012; Styger et al., 2011; Tempère et al., 2018]. In our study, there was no trend in its concentration in Zweigelt and Rondo wines subjected to spontaneous and induced MLF. Its concentration was higher in a few wines in which MLF was spontaneous and in a few wines in which MLF was induced. According to López et al. [2011], spontaneous MLF resulted in a higher content of acetoine in wines, whereas Malherbe et al. [2012] found that acetoin concentration was affected by the bacterial strain used for MLF.

Considering the two quantified furan compounds, grape variety significantly impacted the content of ethyl2-furoate (Table 4$)$. In turn, the concentration of dihydrofuran-2(3H)-one was not influenced by the grape variety, and it is in agreement with the results obtained by Liu et al. [2017]. The yeast strain and yeast $\times$ MLF interaction did not affect ethyl 2-furoate and dihydrofuran-2(3H)-one. Similarly, the yeast did not affect ethyl 2-furoate in studies performed by Callejon et al. [2010]. Concentrations of both furan compounds, ethyl 2-furoate and dihydrofuran-2(3H)-one (butyrolactone), depend on MLF. Butyrolactone is particularly produced during MLF [Tempère et al., 2018]. In our study, the concentration of butyrolactone was higher in most wines subjected to induced MLF. According to López et al. [2011], spontaneous MLF resulted in wines with a higher butyrolactone concentration.

The concentration of 3-(methylsulfanyl)propan-1-ol (Table 4) was not affected by grape variety and MLF, but changes in its values were determined as affected by yeast strain and yeast $\times$ MLF interaction.

Among the analyzed factors, i.e. grape variety, yeast, MLF, yeast $\times$ MLF interaction, only grape variety significantly affected the concentration of 3,5-di-tert-butylphenol (Table 4).

We showed that in the case of Polish wines produced from grape varieties grown in a cold climate, the grape variety was the factor eliciting a significant impact on the highest number of identified volatile compounds, whereas microorganisms used had a lesser effect. In our study, malic acid was not completely reduced during MLF (spontaneous and induced), similarly to the experiment conducted by Lasik-Kurdyś et al. [2018]. Lactic acid has an antimicrobial activity, and at a higher concentration, it can also inhibit LAB. In the case of high-acid musts, a total reduction of malic acid can be impossible [Lasik-Kurdyś et al., 2017].

Figure 1 presents the FTIR spectra of the wines. For more straightforward analysis, discussion, and comparison of the tested wines, the spectra were normalized to the maximum at $3327 \mathrm{~cm}^{-1}$. Table 5 presents all the characteristic bands of wine spectra and appropriate vibrations assigned to the functional groups. All spectra of wines (R1-R5, Z1-Z5, R1 LAB-R5 LAB and Z1 LAB-Z5 LAB) had very intense and similar bands (Figure 1). They exhibited bands characteristic of water and ethanol absorption. The broad peak of 3800-3000 $\mathrm{cm}^{-1}$ results mainly from stretching vibrations in water molecules, $v(-\mathrm{OH})$, and in alcohol or phenol molecules [Basalekou et al., 2019; Geană et al., 2019]. Characteristic bands for vibrations of water molecules are absorption bands with a maximum of about 990 and $1460 \mathrm{~cm}^{-1}$ (stretching and deformation - related to the third overtone of these bands) and characteristic deformation bands with a maximum at about $1600 \mathrm{~cm}^{-1}$ [Geană et al., 2019; Hu et al., 2019]. Alcohol-related absorption bands were observed (for all samples) at $2850-3000 \mathrm{~cm}^{-1}$ with characteristic peaks at 2937 and $2881 \mathrm{~cm}^{-1}$ (Figure 1, Table 5) corresponding to the symmetric and asymmetric stretching vibrations of the $\mathrm{CH}_{2}$ and $\mathrm{CH}_{3}$ groups. The build-up of these bands with a broad and noticeably flattened maximum in the range of 2400-2705 cm-1 could correspond to a combination of C-H stretching vibrations and overtones of these vibrations originating from molecules of ethanol and partly sugar [Basalekou et al., 2019; Geană et al., 2019; Hu et al., 2019]. Vibrations originating from primary alcohols and glycerol with maximum bands at 1087 and $1050 \mathrm{~cm}^{-1}$, respectively, are related to 


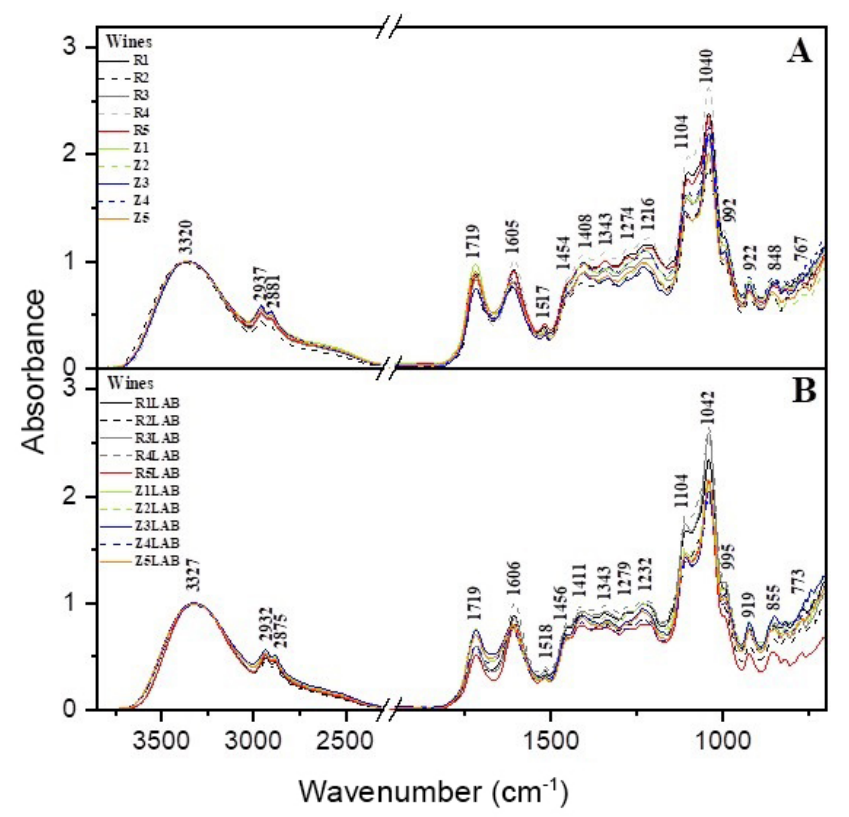

FIGURE 1. Normalized Fourier transform infrared (FTIR) spectra of wines. R1-R5 - Rondo wines, in which alcoholic fermentation (AF) was induced using various yeast strains, and the wines were left to undergo spontaneous malolactic fermentation (MLF); Z1-Z5 - Zweigelt wines, in which $\mathrm{AF}$ was induced using various yeast strains, and the wines were left to undergo spontaneous MLF; R1 LAB-R5 LAB - Rondo wines, in which $\mathrm{AF}$ was induced using various yeast strains (the same strains as in R1-R5 wines), and MLF was carried out by inoculation with lactic acid bacteria; Z1 LAB-Z5 LAB - Zweigelt wines, in which AF was induced using various yeast strains (the same strains as in Z1-Z5 wines), and MLF was carried out by inoculation with lactic acid bacteria.

strong C-O stretching vibrations [Martelo-Vidal et al., 2013]. The bands in the 3000-2800 $\mathrm{cm}^{-1}$ region were most likely due to stretching vibrations of $\mathrm{C}-\mathrm{H}$ bonds of hydrocarbons, $\mathrm{O}-\mathrm{H}$ bonds of carboxylic acids, and asymmetric stretching vibrations of $\mathrm{C}-\mathrm{H}$ bonds of methyl $\left(-\mathrm{CH}_{3}\right)$ groups: polyols (glycerol), free phenolic acids and catechins [Geană et al., 2019].

The vibration area between $1800-1000 \mathrm{~cm}^{-1}$ was characteristic of $\mathrm{C}-\mathrm{OH}$ stretching, $\mathrm{CH}_{3}$ and $\mathrm{CH}_{2}$ deformation, $\mathrm{C}=\mathrm{C}$ stretching, and $\mathrm{C} \equiv \mathrm{N}$ stretching vibrations (Table 5). This area derives from such components as phenols, alcohols, aldehydes, higher alcohols, polyols, acids, sugars, volatile acids and amino acids [Basalekou et al., 2020; Hu et al., 2019; Versari et al., 2014]. The spectral range of about 1850 $-1590 \mathrm{~cm}^{-1}$ was related to the combination of stretching vibrations - $\mathrm{OH},-\mathrm{CH}_{3}$ (first overtone), $-\mathrm{CH}_{2},-\mathrm{CH}$ (first overtone) derived from ethanol [Geană et al., 2019; Martelo-Vidal et al., 2013].

Very interesting vibrations occured in the spectral region of $1580-950 \mathrm{~cm}^{-1}$ (Figure 1). In this area, the vibrations show functional groups characteristic for many wine compounds; therefore, there were more considerable differences between the FTIR spectra obtained in this region. Basically, in the region of $1580-950 \mathrm{~cm}^{-1}$, there were vibrations from phenols of the wines tested. The area between 1460 and $1280 \mathrm{~cm}^{-1}$ was very complex and provided information about stretching vibrations of the carbonyl group $\mathrm{C}=\mathrm{O}$, stretching vibrations $\mathrm{C}=\mathrm{C}, \mathrm{CH}_{2}$, and $\mathrm{C}-\mathrm{H}$ derived from molecules of aldehydes, carboxylic acids, proteins, and esters [Tarantilis et al., 2008].
TABLE 5. The maxima of the Fourier transform infrared absorption bands of wines produced with different yeast and malolactic fermentation (MLF) strategies, with assignment of particular vibrations to the respective wine samples. Spectra registered within the range of $700-3700 \mathrm{~cm}^{-1}$.

\begin{tabular}{|c|c|c|}
\hline $\begin{array}{l}\text { R1-R5 } \\
\text { Z1-Z5 }\end{array}$ & $\begin{array}{l}\text { R1 LAB-R5 LAB } \\
\text { Z1 LAB-Z5 LAB }\end{array}$ & \multirow{2}{*}{ Type and origin of vibrations } \\
\hline \multicolumn{2}{|c|}{ Wavenumber $\left(\mathrm{cm}^{-1}\right)$} & \\
\hline 3320 & 3327 & $v(-\mathrm{OH})$ in carboxylic acids \\
\hline 2937 & 2932 & $v_{w}(-\mathrm{CH})$ of hydrocarbons \\
\hline 2881 & 2875 & $v_{\mathrm{m}}\left(-\mathrm{CH}_{3}\right)$ \\
\hline 1719 & 1719 & $v_{\mathrm{m}}(-\mathrm{C}=\mathrm{O})$ \\
\hline 1605 & 1606 & $\delta(-\mathrm{OH})$ and $v(\mathrm{C}=\mathrm{C})$ \\
\hline 1517 & 1518 & $v(\mathrm{C}=\mathrm{C})$ and $v(\mathrm{C}-\mathrm{N})$ \\
\hline 1454 & 1456 & $v(\mathrm{C}=\mathrm{C}), \delta\left(-\mathrm{CH}_{3}\right)$ \\
\hline 1408 & 1411 & $\delta_{\mathrm{m}}\left(-\mathrm{CH}_{2}\right)$ and $\delta(-\mathrm{CH})$ \\
\hline 1343 & 1343 & $v(\mathrm{C}=\mathrm{C}), \delta\left(-\mathrm{CH}_{2}\right)$ \\
\hline 1274 & 1279 & $\delta\left(-\mathrm{CH}_{2}-\right)$ \\
\hline 1216 & 1232 & $v_{m}(-\mathrm{C}-\mathrm{O})$ or $\delta_{\mathrm{m}}\left(-\mathrm{CH}_{2}-\right)$ \\
\hline 1104 & 1104 & $\begin{array}{c}v_{\mathrm{st}}(-\mathrm{C}-\mathrm{O}) \text { and } v_{\mathrm{w}}(\mathrm{O}-\mathrm{H}) \\
\text { second overtones }\end{array}$ \\
\hline 1040 & 1042 & $v_{\mathrm{m}}(-\mathrm{C}-\mathrm{O})$ \\
\hline 992 & 995 & \\
\hline 922 & 919 & $\begin{array}{c}\delta_{\mathrm{w}}(-\mathrm{HC}=\mathrm{CH}-, \text { trans- }) \\
\text { out-of-plane }\end{array}$ \\
\hline 848 & 855 & $\begin{array}{c}\delta\left(-\left(\mathrm{CH}_{2}\right)_{\mathrm{n}}-\text { and }-\mathrm{HC}=\mathrm{CH}-(\text { cis- })\right. \\
(\text { scissor })\end{array}$ \\
\hline 767 & 773 & \\
\hline
\end{tabular}

$v$ - stretching vibrations; $\delta$ - deformation vibrations; st - strong; m - medium; w - weak; R1-R5 - Rondo wines, in which alcoholic fermentation (AF) was induced using various yeast strains, and the wines were left to undergo spontaneous MLF; Z1-Z5 - Zweigelt wines, in which $\mathrm{AF}$ was induced using various yeast strains, and the wines were left to undergo spontaneous MLF; R1 LAB-R5 LAB - Rondo wines, in which $\mathrm{AF}$ was induced using various yeast strains (the same strains as in R1-R5 wines), and MLF was carried out by inoculation with lactic acid bacteria; Z1 LAB-Z5 LAB - Zweigelt wines, in which AF was induced using various yeast strains (the same strains as in Z1-Z5 wines), and MLF was carried out by inoculation with lactic acid bacteria

The ester bands showed very characteristic peaks at about 1460-1400 cm-1 [Geană et al., 2019; Tarantilis et al., 2008]. It is also worth emphasizing the bands with maxima of around 1232, 1110-1100, and 1070-990 $\mathrm{cm}^{-1}$, which correspond to $\mathrm{C}-\mathrm{O}$ and $\mathrm{O}-\mathrm{H}$ stretching vibrations (second overtone) derived from sugars and organic acids [Ferreiro-González et al., 2019; Hu et al., 2019; Martelo-Vidal et al., 2013; Tarantilis et al., 2008].

Spectral analysis showed differences in the area for groups associated with alcohols, esters, and acids. It was most likely related to the grape varieties used, however, the main constituents formed during fermentation could also explain the difference. The wines marked as LAB (induced MLF) in the area mentioned above differed from the wines which were left to undergo spontaneous MLF. 


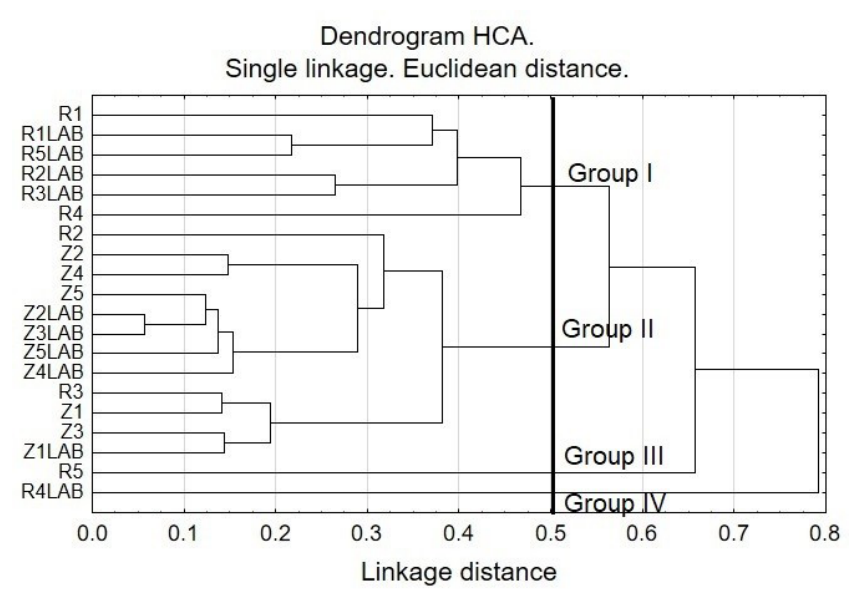

FIGURE 2. Dendrogram of hierarchical cluster analysis (HCA) of wines based on the Fourier transform infrared (FTIR) spectral data for wavenumber range of 1580-950 $\mathrm{cm}^{-1}$. R1-R5 - Rondo wines, in which alcoholic fermentation (AF) was induced using various yeast strains, and the wines were left to undergo spontaneous malolactic fermentation (MLF); Z1-Z5 - Zweigelt wines, in which AF was induced using various yeast strains, and the wines were left to undergo spontaneous MLF; $\mathrm{R} 1 \mathrm{LAB}-\mathrm{R} 5 \mathrm{LAB}$ - Rondo wines, in which $\mathrm{AF}$ was induced using various yeast strains (the same strains as in R1-R5 wines), and MLF was carried out by inoculation with lactic acid bacteria; Z1 LAB-Z5 LAB - Zweigelt wines, in which $\mathrm{AF}$ was induced using various yeast strains (the same strains as in Z1-Z5 wines), and MLF was carried out by inoculation with lactic acid bacteria.

HCA was performed on FTIR spectra to identify similarities or dissimilarities between the considered samples of wine [Brereton, 2003]. Figure 2 shows the dendrogram obtained from the 20 wine samples. Considering the cut-off of 0.5 dissimilarity units, four clusters were distinguished. The first cluster (Group I) aggregated on the far-left arm of the dendrogram and was formed by six wine samples, while the second cluster (Group II) was the biggest cluster and comprised all samples of Zweigelt wine and two samples of Rondo $\mathrm{R} 2$ and R3. This result suggests these wines have physicochemical properties more similar than the others. The last two Rondo wine samples - R5 and R4 LAB aggregated in third and fourth clusters. The hierarchical cluster analysis showed that the placement of the wine samples on the dendrogram depended on the grape variety and type of MLF (spontaneous or induced).

To more precisely discriminate the relationship between the investigated wines, the $1580-950 \mathrm{~cm}^{-1}$ bands were selected as their characteristic spectral fingerprint. The PCA was set as the primary choice for analysis. PCA transforms the original high-dimensional variables into the new low-dimensional variables [Abdi \& Williams, 2010; Xu et al., 2006]. According to the Scree test criterion, we selected two main components for analysis. Figure 3 shows a two-dimensional scatter plot of the principal components (PC1 and PC2) obtained from the FTIR spectra of different wine samples. The PC1 was the most critical and explained $98.3 \%$ of the variance; the second principal component contributed $1.1 \%$ to the variance. The first two principal components explained $99.4 \%$ of the variance, and only $0.6 \%$ of the information was lost. It indicates that the first two principal components expressed $99.4 \%$ of all

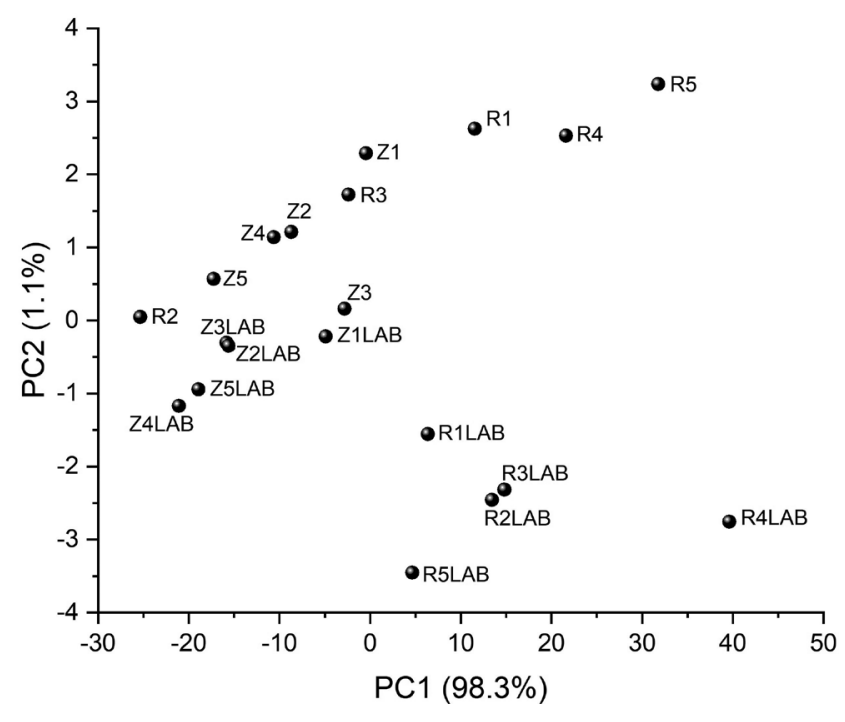

FIGURE 3. The scattered scores plot of principal component analysis (PCA) based on Fourier transform infrared (FTIR) spectra fingerprint of wines. R1-R5 - Rondo wines, in which alcoholic fermentation (AF) was induced using various yeast strains, and the wines were left to undergo spontaneous MLF; Z1-Z5 - Zweigelt wines, in which AF was induced using various yeast strains, and the wines were left to undergo spontaneous malolactic fermentation (MLF); R1 LAB-R5 LAB - Rondo wines, in which $\mathrm{AF}$ was induced using various yeast strains (the same strains as in R1-R5 wines), and MLF was carried out by inoculation with lactic acid bacteria; Z1 LAB-Z5 LAB - Zweigelt wines, in which AF was induced using various yeast strains (the same strains as in Z1-Z5 wines), and MLF was carried out by inoculation with lactic acid bacteria.

the information. Figure 3 and Figure S3 (supplementary materials) show the relative location of the FTIR spectra in the two-dimensional graph. The location was related to their similarity distance shown by HCA (Figure 2). For example, the close location of Zweigelt wines in the PCA plot was related to their close locations in the HCA graph. Samples R4 LAB and R5, which did not clustered with the other Rondo wines (Figure 2), were obviously distinguished from other samples shown in the two-dimensional PCA graph (Figure 3). Samples of the Zweigelt variety distinguished one another in the same category, as shown on the groupedscatter plot for all samples (Figure S3). These samples differed only to a slight extent. As it is known, the scatter plot does not classify the objects in every level of distance as accurately as the clustering graph. Still, it can reflect the relationships between the investigated wines. Therefore, the use of complementary analytical methods provides researchers profound knowledge of the wine differences.

\section{CONCLUSIONS}

Among the factors studied, the grape variety significantly affected most volatile compounds, most alcohols and acids, and half of the esters. The yeast strains selected in this study had no significant effect on the concentration of either any ester or any acid. MLF significantly influenced the concentration of almost half of esters and acids. Among forty-six quantified compounds, only three were affected by yeast $\times$ MLF interaction. Knowledge of the influence of grape, yeast, MLF, and yeast $\times$ MLF interaction on wine aroma may help 
producers make informed decisions. Further investigations into the effects of the wine matrix on the production of volatile compounds by microorganisms are also required.

The results of FTIR measurements showed differences, most remarkable in the range of 1750-1500 and below $1500 \mathrm{~cm}^{-1}$. These differences are mainly related to various concentrations of volatile compounds, such as alcohols, acids, and esters, in the wines. The results obtained in the experiment may contribute to the expansion of the wine database.

\section{SUPPLEMENTARY MATERIALS}

The following are available online at http://journal.pan. olsztyn.pl/Impact-of-Grape-Variety-Yeast-and-MalolacticFermentation-on-Volatile-Compounds-and,145665,0,2.html. The concentrations of malic and lactic acids in the final wines; chromatograms of volatile compounds of Zweigelt and Rondo wines, and PCA results.

\section{CONFLICT OF INTERESTS}

Authors declare no conflict of interests.

\section{ORCID IDs}

T. Czernecki https://orcid.org/0000-0002-3277-2326

A. Matwijczuk https://orcid.org/0000-0003-2630-120X

A. Niemczynowicz https://orcid.org/0000-0002-4370-3326

B. Sosnowska https://orcid.org/0000-0001-9719-9359

A. Stój https://orcid.org/0000-0003-1824-2051

\section{REFERENCES}

1. Azzolini, M., Fedrizzi, B., Tosi, E., Finato, F., Vagnoli, P., Scrinzi, C., Zapparoli, G. (2012). Effects of Torulaspora delbrueckii and Sacharomyces cerevisiae mixed cultures on fermentation and aroma of Amarone wine. European Food Research and Technology, 235, 303-313.

https://doi.org/10.1007/s00217-012-1762-3

2. Abdi, H., Williams, L.J. (2010). Principal component analysis. Wiley Interdisciplinary Reviews: Computational Statistics, 2(4), 433-459.

https://doi.org/10.1002/wics.101

3. Abrahamse, C.E., Bartowsky, E.J. (2012). Timing of malolactic fermentation inoculation in Shiraz grape must and wine: influence on chemical composition. World Journal of Microbiology and Biotechnology, 28(1), 255-265.

https://doi.org/10.1007/s11274-011-0814-3

4. Antalick, G., Perello, M.C., de Revel, G. (2013). Co-inoculation with yeast and LAB under winery conditions: modification of the aromatic profile of Merlot wines. South African Journal of Enology and Viticulture, 34(2), 223-232.

https://doi.org/10.21548/34-2-1098

5. Basalekou, M., Kallithraka, S., Tarantilis, P.A., Kotseridis, Y., Pappas, C. (2019). Ellagitannins in wines: Future prospects in methods of analysis using FT-IR spectroscopy. LWT - Food Science and Technology, 101, 48-53.

https://doi.org/10.1016/j.Iwt.2018.11.017
6. Basalekou, M., Pappas, C., Tarantilis, P.A., Kallithraka, S. (2020). Wine authenticity and traceability with the use of FTIR. Beverages, 6(2), art. no. 30. https://doi.org/10.3390/beverages6020030

7. Blanco, P., Mirás-Avalos, J.M., Pereira, E., Fornos, D., Orriols, I. (2014). Modulation of chemical and sensory characteristics of red wine from Mencía by using indigenous Saccharomyces cerevisiae yeast strains. Journal International des Sciences de la Vigne et du Vin, 48(1), 63-74.

https://doi.org/10.20870/oeno-one.2014.48.1.1659

8. Brizuela, N.S., Bravo-Ferrada, B.M., Pozo-Bayón, M.Á., Semorile, L., Tymczyszyn, E.E. (2018). Changes in the volatile profile of Pinot noir wines caused by Patagonian Lactobacillus plantarum and Oenococcus oeni strains. Food Research International, 106, 22-28.

https://doi.org/10.1016/j.foodres.2017.12.032

9. Brereton, R.G. (2003). Pattern recognition. Chapter 4. In R.G. Brereton (Ed.) Chemometrics: Data Analysis for the Laboratory and Chemical Plant. John Wiley \& Sons, Chichester, pp. 183-269.

https://doi.org/10.1002/0470863242.ch4

10. Callejon, R.M., Clavijo, A., Ortigueira, P., Troncoso, A.M., Paneque, P., Morales, M.L. (2010). Volatile and sensory profile of organic red wines produced by different selected autochthonous and commercial Saccharomyces cerevisiae strains. Analytica Chimica Acta, 660(1-2), 68-75. https://doi.org/10.1016/j.aca.2009.09.040

11. Cañas, P.M.I., Pérez-Martín, F., Romero, E.G., Prieto, S.S., Herreros, M.D.L.L.P. (2012). Influence of inoculation time of an autochthonous selected malolactic bacterium on volatile and sensory profile of Tempranillo and Merlot wines. International Journal of Food Microbiology, 156(3), 245-254. https://doi.org/10.1016/j.ijfoodmicro.2012.03.033

12. Cioch-Skoneczny, M., Grabowski, M., Satora, P., Skoneczny, S., Klimczak, K. (2021). The use of yeast mixed cultures for deacidification and improvement of the composition of cold climate grape wines. Molecules, 26(9), art. no. 2628. https://doi.org/10.3390/molecules26092628

13. Costello, P.J., Francis, I.L., Bartowsky, E.J. (2012). Variations in the effect of malolactic fermentation on the chemical and sensory properties of Cabernet Sauvignon wine: Interactive influences of Oenococcus oeni strain and wine matrix composition. Australian Journal of Grape and Wine Research, 18(3), 287-301. https://doi.org/10.1111/j.1755-0238.2012.00196.x

14. Diez-Ozaeta, I., Lavilla, M., Amárita, F. (2021). Wine aroma profile modification by Oenococcus oeni strains from Rioja Alavesa region: selection of potential malolactic starters. International Journal of Food Microbiology, 356, art. no. 109324. https://doi.org/10.1016/j.ijfoodmicro.2021.109324

15. Duarte, W.F., Dias, D.R., Oliveira, J.M., Teixeira, J.A., De Almeida e Silva, J.B., Schwan, R.F. (2010). Characterization of different fruit wines made from cacao, cupuassu, gabiroba, jaboticaba and umbu. LWT - Food Science and Technology, 43(10), 1564-1572. https://doi.org/10.1016/j.Iwt.2010.03.010

16. Englezos, V., Rantsiou, K., Cravero, F., Torchio, F., Giacosa, S., Ortiz-Julien, A., Gerbi, V., Rolle, L. Cocolin, L. (2018). Volatile profiles and chromatic characteristics of red wines produced 
with Starmerella bacillaris and Saccharomyces cerevisiae. Food Research International, 109, 298-309.

https://doi.org/10.1016/j.foodres.2018.04.027

17. Ferreiro-González, M., Ruiz-Rodríguez, A., Barbero, G.F., Ayuso, J., Álvarez, J.A., Palma, M., Barroso, C.G. (2019). FT-IR, Vis spectroscopy, color and multivariate analysis for the control of ageing processes in distinctive Spanish wines. Food Chemistry, 277, 6-11. https://doi.org/10.1016/j.foodchem.2018.10.087

18. Gammacurta, M., Marchand, S., Albertin, W., Moine, V., de Revel, G. (2014). Impact of yeast strain on ester levels and fruity aroma persistence during aging of Bordeaux red wines. Journal of Agricultural and Food Chemistry, 62(23), 5378-5389. https://doi.org/10.1021/jf500707e

19. Gammacurta, M., Marchand, S., Moine, V., de Revel, G. (2017). Influence of different yeast/lactic acid bacteria combinations on the aromatic profile of red Bordeaux wine. Journal of the Science of Food and Agriculture, 97(12), 4046-4057.

https://doi.org/10.1002/jsfa.8272

20. Geană, E.I., Ciucure, C.T., Apetrei, C., Artem, V. (2019). Application of spectroscopic UV-Vis and FT-IR screening techniques coupled with multivariate statistical analysis for red wine authentication: Varietal and vintage year discrimination. Molecules, 24(22), art. no. 4166.

https://doi.org/10.3390/molecules24224166

21. Hu, X.Z., Liu, S.Q., Li, X.H., Wang, C.X., Ni, X.L., Liu, X., Wang, Y, Liu, Y., Xu, C.H. (2019). Geographical origin traceability of Cabernet Sauvignon wines based on infrared fingerprint technology combined with chemometrics. Scientific Reports, 9, art. no. 8256 .

https://doi.org/10.1038/s41598-019-44521-8

22. Jørgensen, U., Hansen, M., Christensen, L.P., Jensen, K., Kaack, K. (2000). Olfactory and quantitative analysis of aroma compounds in elder flower (Sambucus nigra L.) drink processed from five cultivars. Journal of Agricultural and Food Chemistry, 48(6), 2376-2383. https://doi.org/10.1021/jf000005f

23. Lasik-Kurdyś, M., Majcher, M., Nowak, J. (2018). Effects of different techniques of malolactic fermentation induction on diacetyl metabolism and biosynthesis of selected aromatic esters in cool-climate grape wines. Molecules, 23(10), art. no. 2549. https://doi.org/10.3390/molecules23102549

24. Lasik-Kurdýs, M., Gumienna, M., Nowak, J. (2017). Influence of malolactic bacteria inoculation scenarios on the efficiency of the vinification process and the quality of grape wine from the Central European region. European Food Research and Technology, 243, 2163-2173.

https://doi.org/10.1007/s00217-017-2919-x

25. Liu, J., Arneborg, N., Toldam-Andersen, T.B., Petersen, M.A., Bredie, W.L. (2017). Effect of sequential fermentations and grape cultivars on volatile compounds and sensory profiles of Danish wines. Journal of the Science of Food and Agriculture, 97(11), 3594-3602.

https://doi.org/10.1002/jsfa.8218

26. López, R., López-Alfaro, I., Gutiérrez, A.R., Tenorio, C., Garijo, P., González-Arenzana, L., Santamaría, P. (2011). Malolactic fermentation of Tempranillo wine: contribution of the lactic acid bacteria inoculation to sensory quality and chemical composition. International Journal of Food Science \& Technology, 46(11), 2373-2381.

https://doi.org/10.1111/j.1365-2621.2011.02759.x
27. Malherbe, S., Tredoux, A.G., Nieuwoudt, H.H., du Toit, M. (2012). Comparative metabolic profiling to investigate the contribution of $O$. oeni MLF starter cultures to red wine composition. Journal of Industrial Microbiology \& Biotechnology, 39(3), 477-494. https://doi.org/10.1007/s10295-011-1050-4

28. Mallouchos, A., Loukatos, P., Bekatorou, A., Koutinas, A., Komaitis, M. (2007). Ambient and low temperature winemaking by immobilized cells on brewer's spent grains: Effect on volatile composition. Food Chemistry, 104(3), 918-927. https://doi.org/10.1016/j.foodchem.2006.12.047

29. Martelo-Vidal, M.J., Domínguez-Agis, F., Vázquez, M. (2013). Ultraviolet/visible/near-infrared spectral analysis and chemometric tools for the discrimination of wines between subzones inside a controlled designation of origin: A case study of Rías Baixas. Australian Journal of Grape and Wine Research, 19(1), 62-67. https://doi.org/10.1111/ajgw.12003

30. Mendes, B., Gonçalves, J., Câmara, J.S. (2012). Effectiveness of high-throughput miniaturized sorbent- and solid phase microextraction techniques combined with gas chromatography-mass spectrometry analysis for a rapid screening of volatile and semi-volatile composition of wines - a comparative study. Talanta, 88, 79-94. https://doi.org/10.1016/j.talanta.2011.10.010

31. Ruocco, S., Perenzoni, D., Angeli, A., Stefanini, M., Rühl, E., Patz, C.D., Mattivi, F., Rauhut, D., Vrhovsek, U. (2019). Metabolite profiling of wines made from disease-tolerant varieties. European Food Research and Technology, 245(9), 2039-2052. https://doi.org/10.1007/s00217-019-03314-z

32. Shimoda, M., Shigematsu, H., Shiratsuchi, H., Osajima, Y. (1995). Comparison of the odor concentrates by SDE and adsorptive column method from green tea infusion. Journal of Agricultural and Food Chemistry, 43(6), 1616-1620. https://doi.org/10.1021/jf00054a037

33. Song, S., Tanga, Q., Hayat, K., Karangwa, E., Zhang, X., Xiao, Z. (2014). Effect of enzymatic hydrolysis with subsequent mild thermal oxidation of tallow on precursor formation and sensory profiles of beef flavours assessed by partial least squares regression. Meat Science, 96(3), 1191-1200. https://doi.org/10.1016/j.meatsci.2013.11.008

34. Stój, A., Czernecki, T., Domagała, D., Targoński, Z. (2017a). Comparative characterization of volatile profiles of French, Italian, Spanish, and Polish red wines using headspace solid-phase microextraction/gas chromatography-mass spectrometry. International Journal of Food Properties, 20(sup1), S830-S845. https://doi.org/10.1080/10942912.2017.1315590

35. Stój, A., Czernecki, T., Domagala, D., Targoński, Z. (2017b). Application of volatile compound analysis for distinguishing between red wines from Poland and from other European countries. South African Journal of Enology and Viticulture, 38(2), 245-263. https://doi.org/10.21548/38-2-2079

36. Stój, A. (2020a). Influence of yeast and lactic acid bacteria on the content of volatile compounds and other oenological parameters of wines. Postęy Mikrobiologii - Advancements of Microbiology, 59(2), 167-178 (in Polish; English abstract). https://doi.org/10.21307/PM-2020.59.2.013

37. Stój, A., Kapusta, I., Domagała, D. (2020b). Classification of red wines produced from Zweigelt and Rondo grape varieties based on the analysis of phenolic compounds by UPLC-PDA-MS/ MS. Molecules, 25(6), art. no. 1342. https://doi.org/10.3390/molecules25061342 
38. Sumby, K.M., Jiranek, V., Grbin, P.R. (2013). Ester synthesis and hydrolysis in an aqueous environment, and strain specific changes during malolactic fermentation in wine with Oenococcus oeni. Food Chemistry, 141(3), 1673-1680.

https://doi.org/10.1016/j.foodchem.2013.03.087

39. Styger, G., Prior, B., Bauer, F.F. (2011). Wine flavor and aroma. Journal of Industrial Microbiology \& Biotechnology, 38(9), 1145-1159.

https://doi.org/10.1007/s10295-011-1018-4

40. Tarantilis, P.A., Troianou, V.E., Pappas, C.S., Kotseridis, Y.S. Polissiou, M.G. (2008). Differentiation of Greek red wines on the basis of grape variety using attenuated total reflectance Fourier transform infrared spectroscopy. Food Chemistry, 111 (1), 192-196. https://doi.org/10.1016/j.foodchem.2008.03.020

41. Tempère, S., Marchal, A., Barbe, J.C., Bely, M., Masneuf-Pomarede, I., Marullo, P., Albertin, W. (2018). The complexity of wine: clarifying the role of microorganisms. Applied Microbiology and Biotechnology, 102(9), 3995-4007.

https://doi.org/10.1007/s00253-018-8914-8

42. Tristezza, M., di Feo, L., Tufariello, M., Grieco, F., Capozzi, V., Spano, G., Mita, G. (2016). Simultaneous inoculation of yeasts and lactic acid bacteria: Effects on fermentation dynamics and chemical composition of Negroamaro wine. LWT - Food Science and Technology, 66, 406-412.

https://doi.org/10.1016/j.Iwt.2015.10.064

43. Tufariello, M., Chiriatti, M.A., Grieco, F., Perrotta, C., Capone, S., Rampino, P., Tristezza, M., Mita, G., Grieco, F. (2014). Influence of autochthonous Saccharomyces cerevisiae strains on volatile profile of Negroamaro wines. LWT - Food Science and Technology, 58(1), 35-48.

https://doi.org/10.1016/j.lwt.2014.03.016
44. Versari, A., Laurie, V.F., Ricci, A., Laghi, L., Parpinello, G.P. (2014). Progress in authentication, typification and traceability of grapes and wines by chemometric approaches. Food Research International, 60, 2-18.

https://doi.org/10.1016/j.foodres.2014.02.007

45. Vilanova, M., Cortés, S., Santiago, J.L., Martínez, C., Fernández, E. (2007). Aromatic compounds in wines produced during fermentation: effect of three red cultivars. International Journal of Food Properties, 10(4), 867-875.

https://doi.org/10.1080/10942910601161615

46. Welke, J.E., Manfroi, V., Zanus, M., Lazzarotto, M., Zini, C.A. (2012). Characterization of the volatile profile of Brazilian Merlot wines through comprehensive two dimensional gas chromatography time-of-flight mass spectrometric detection. Journal of Chromatography A, 1226, 124-139.

https://doi.org/10.1016/j.chroma.2012.01.002

47. Wojdyło, A., Samoticha, J., Nowicka, P., Chmielewska, J. (2018). Characterisation of (poly)phenolic constituents of two interspecific red hybrids of Rondo and Regent (Vitis vinifera) by LC-PDA-ESI-MS QTof. Food Chemistry, 239, 94-101. https://doi.org/10.1016/j.foodchem.2017.06.077

48. Xu, C.J., Liang, Y.Z., Chau, F.T., Vander Heyden, Y. (2006). Pretreatments of chromatographic fingerprints for quality control of herbal medicines. Journal of Chromatography A, 1134(1-2), 253-259.

https://doi.org/10.1016/j.chroma.2006.08.060

49. Zhu, F., Du, B., Li, J. (2016). Aroma compounds in wine. In A. Morata, I. Loira (Eds.), Grape and Wine Biotechnology, Books on Demand GmbH, Norderstedt, Germany, pp. 273-283. https://doi.org/10.5772/65102 
